\title{
1 Frequency dependent growth of bacteria in living materials
}

2 Daniel D. Lewis ${ }^{1,2,}$, , Ting Gong ${ }^{1, *}$, Yuanwei $X u^{3}$, Cheemeng Tan ${ }^{1,+}$

$3{ }^{1}$ Department of Biomedical Engineering, University of California Davis, Davis, CA

$4 \quad{ }^{2}$ Integrative Genetics and Genomics, University of California Davis, Davis, CA

$5 \quad{ }^{3}$ Department of Biomedical Engineering, Peking University, Beijing, China

$6 \quad{ }^{+}$Corresponding author (cmtan@ucdavis.edu)

$7 \quad$ *Equal contribution

\section{Abstract}

The fusion of living bacteria and man-made materials represents a new frontier in medical and biosynthetic technology. However, the principles of bacterial signal

12 processing inside three dimensional and fluctuating environments of synthetic materials

13 remain elusive. Here, we study bacterial growth in a three-dimensional hydrogel. We

14 find that bacteria expressing an antibiotic resistance module can take advantage of

15 ambient kinetic disturbances to improve growth while encapsulated. We show that these

16 changes in bacterial growth are specific to disturbance frequency and hydrogel density.

17 This remarkable specificity is consistent with stochastic resonance theory, which we

18 leverage to explain how bacteria can integrate spatial and temporal information to

19 control growth. This research provides a quantitative foundation for the control of living

20 materials and a systematic framework towards understanding bacterial information

21 processing in three-dimensional environments. 


\section{Introduction}

The integration of designer microbes with synthetic materials has birthed a new class of customizable, environmentally-responsive living materials (Balasubramanian et al., 2019; Cao et al., 2017; Gilbert et al., 2021; Gonzalez et al., 2020; Hay et al., 2018; Manjula-Basavanna et al., 2021). Genetic circuits inside bacteria can be designed to produce enzymatic or structural components based on intra- or extra-cellular cues. Through expression regulation mediated by genetic circuits, designer microbes can control self-assembly (Cao et al., 2017) and adaptation (Gilbert et al., 2021; Gonzalez et al., 2020; Hay et al., 2018) in synthetic materials. Designer microbes are often incorporated into hydrogels to form engineered living materials (Chyzy and PlonskaBrzezinska, 2020). Hydrogels' physical properties, such as stiffness, density, and viscosity, play an important role in the metabolic activity and growth rate of encapsulated cells (Gilbert et al., 2021; Manjula-Basavanna et al., 2021). While the interactions between hydrogels and cellular growth can be exploited to control selfassembly (Cao et al., 2017), they can also impart metabolic heterogeneity. Metabolic heterogeneity of encapsulated cells in synthetic materials is known to impede their growth and genetic circuit activity (Alvarez et al., 2009; Pabst et al., 2016; Wessel et al., 2013). To expand the utility and range of biological functions in engineered living materials, there is a critical need to understand and control the growth of synthetically encapsulated microbes. are capable of integrating complex environmental inputs into decisions that reshape cellular physiology (Dufrene and Persat, 2020; Taute et al., 2015). However, these studies have also revealed gaps in our understanding of how bacteria integrate temporally and spatially heterogeneous signals into beneficial phenotypic changes (Kandemir et al., 2018; Sanfilippo et al., 2019). There have been explorations of the connections between spatial structure and information processing in microbial colonies (Alnahhas et al., 2019; Bittihn et al., 2020; Dal Co et al., 2020; Gupta et al., 2020; Kong et al., 2017; Kussell and Leibler, 2005; van Vliet et al., 2018; Zhang et al., 2021), but these studies have not addressed the information processing of microbes embedded in 
three-dimensional synthetic materials. Along this line, studies using engineered cells suggest that phenomena like Turing patterns may facilitate collective decision-making based on spatial and temporal information (Cao et al., 2016; Karig et al., 2018). Do unique information-processing principles govern microbial behavior in three-dimensional materials? If so, could those principles be used to control the growth of microbes encapsulated in synthetic materials?

Here, we study the capacity of bacteria to capitalize on rapid periodic disturbances in a synthetic hydrogel. Rapid periodic disturbances are common in complex 3D environments that are key targets for engineered living materials (Nguyen et al., 2018), such as cardiac tissue, airways, and intestines. We use an antibiotic resistance gene to explore how the metabolic trade-off of a synthetic genetic module affects bacterial growth in a spatially heterogeneous and temporally fluctuating environment. This work uncovers a counterintuitive phenomenon where the frequency of rapid periodic disturbances optimizes the growth of antibiotic-resistant bacteria encapsulated in hydrogel. A mathematical model recapitulates this frequencydependent optimization of growth by proposing that cells can leverage stochastic resonance (McDonnell and Abbott, 2009; Paulsson et al., 2000; Zeng et al., 2009) to integrate spatial and temporal information to survive in synthetic materials.

\section{Results}

Bacteria exhibit fluctuating ATP metabolism in periodically perturbed hydrogel

Spatial heterogeneity caused by hydrogel encapsulation can disrupt cellular metabolism underlying signal propagation (Shao et al., 2017). However, it was unknown whether these disruptions in metabolism could prevent a cell from responding to rapid fluctuations in its environment. To address this unknown, we grew bacteria in a culture system where we could modulate the heterogeneity of the media, periodically perturb the cells, and measure the subsequent changes in intracellular signals (Fig. 1A).

82 Escherichia coli DH5 $\alpha$ was used as a basal strain, cultured in freshly-prepared LB (Luria 83 Broth) media and hydrogel. Encapsulation conditions were controlled by dissolving 84 hydrogel in media by autoclaving, then storing the media at $37^{\circ} \mathrm{C}$ until use (Methods- 
Figure 1

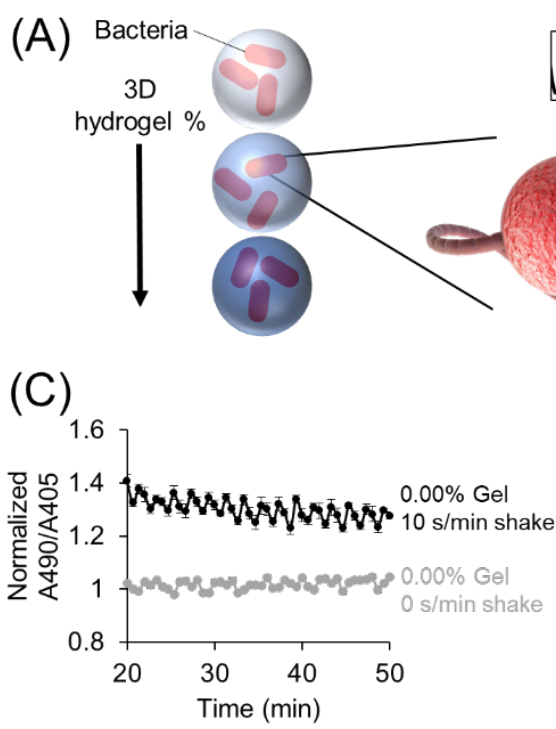

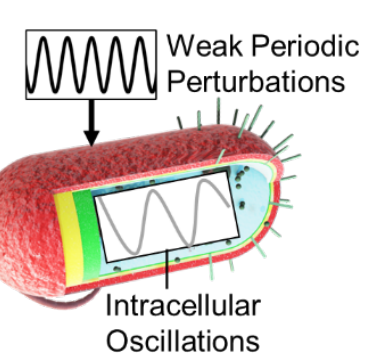

(D)

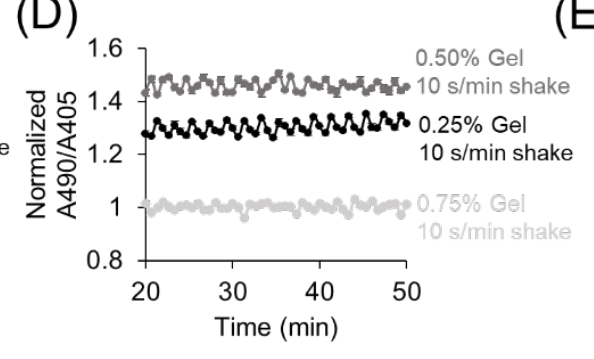

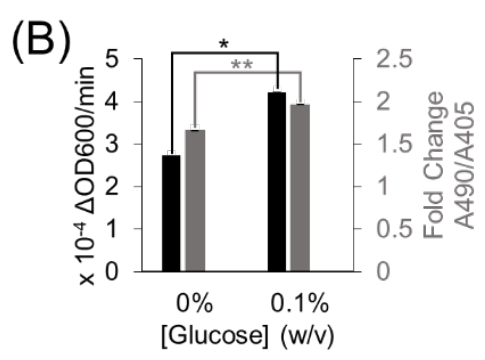

(E)

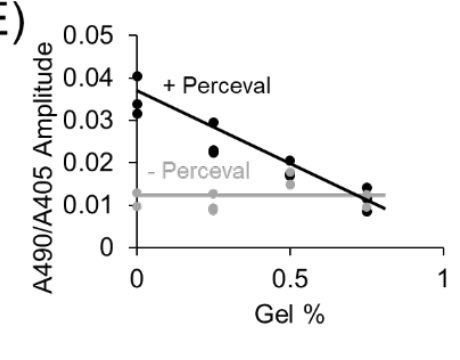

Figure 1: Periodic environment perturbations can propagate to intrabacterial metabolite oscillations despite hydrogel encapsulation.

(A) Cartoon depicting bacteria encapsulated in varying densities of hydrogel being subjected to external kinetic perturbation. Experiment assays whether external perturbations propagate to internal fluctuations in cellular metabolites.

(B) Bacterial growth rate (black bar) and their perceval expression (grey bar) in M9 minimal medium ( 0 and $0.1 \%$ glucose, respectively). Difference between bacterial growth rates is significant ( $p$ value $<0.05$, indicated by asterisk). Difference between perceval values is significant $(p$-value $<0.005$, indicated by two asterisks). Bar height represents mean values, and error bars represent standard error of the mean. Mean and SEM are calculated using four biological replicates.

(C) Intrabacterial ATP/ADP ratio over time with 0 or $10 \mathrm{~s} / \mathrm{min}$ shaking. Absorbance at $490 \mathrm{~nm}$ to absorbance at $405 \mathrm{~nm}$ ratio represents ATP/ADP ratio. Basal fluctuation in A490/A405 without shaking is likely caused by the mechanical movement of the platereader to read the samples. Points represent mean values, and error bars represent standard error of the mean. Mean and SEM are calculated using four biological replicates.

(D) Intrabacterial ATP/ADP ratio at different hydrogel densities $(0.25 \%-0.75 \%$ gel). Absorbance at 490 $\mathrm{nm}$ to absorbance at $405 \mathrm{~nm}$ ratio suggests that periodic perturbation of hydrogel encapsulated cells can propagate to changes in intracellular metabolites. Points represent mean values, and error bars represent standard error of the mean. Mean and SEM are calculated using four biological replicates. (E) Amplitude of ATP/ADP fluctuations in E. coli DH5a cells (grey line) and Perceval cells (black line) at different gel concentrations $(0-0.75 \%)$. Results show that increasing hydrogel concentration reduces amplitude of ATP/ADP ratio fluctuation. Points represent individual replicates, line highlights trend. At least two replicates per condition.

To monitor changes in intracellular metabolites, bacteria were grown expressing

87 the metabolic reporter known as Perceval (Berg et al., 2009) (Methods- Section B\&C). growth rate (Fig. 1B, black bar, $p$-value: 0.02 ) correlated with an increased ratio of 
91 Since the ratio of A490/A405 corresponds to the ATP/ADP ratio, these results suggest

92 that the Perceval reporter can serve as a proxy for cellular bioactivity. These results are 93 consistent with the previous use of ATP as a reporter of metabolic activity (Lopatkin et

94 al., 2019).

Next, bacteria expressing Perceval were cultured in a periodically-shaken environment and changes in the ATP to ADP ratio were measured over time at different gel concentrations. In cultures shaken for $10 \mathrm{~s} / \mathrm{min}$, oscillations occurred in the A490/A405 ratio (Fig. 1C black). Some A490/A405 fluctuation was observed in the absence of shaking (Fig. 1C grey), but this is likely due to the mechanical movement required to measure different wells every two minutes. When hydrogel-encapsulated bacteria were shaken for $10 \mathrm{~s} / \mathrm{min}$, oscillation in the A490/A405 ratios was also recorded (Fig. 1D black, dark grey). These results challenge the assumption that heterogeneous conditions eliminate the dynamic responsiveness of intracellular metabolites to environmental conditions. High concentrations of gel were able to repress oscillations in the A490/A405 ratio (Fig. 1D light grey). These results suggest that sufficiently concentrated environmental hydrogel can dampen short-term cellular metabolic responses. A power spectral analysis (Fig. 1E, Supp. Fig. $12^{\text {nd }}$ Column) revealed that Perceval-expressing cells at $0.25 \%$ gel experienced significantly higher oscillation amplitude ( $p$-value: 0.01) than background fluctuations in A490/A405 measurements. We observed similar periodicity in bacteria expressing a luminescent reporter under periodic and heterogeneous conditions (Supp. Fig. 2, Methods- Section D). These results demonstrate that rapidly oscillating physical perturbation of hydrogel113 encapsulated bacteria can propagate to intracellular metabolic changes (Fig. 1C-E, Supp Fig 1-2).

Bacterial gene expression and growth rate can be controlled by exogenous hydrogel

To confirm that the hydrogel used in this study imparts heterogeneity on growing 117 cells, bacterial growth in a 3D hydrogel was measured. Bacterial density was recorded 118 for two hours at a range of different hydrogel concentrations (Supp Fig. 3A-D top rows). 119 Bacterial density at two hours was used to compare growth between cultures, serving 120 as a surrogate reporter of cellular bioactivity (Fig. 2A). The two-hour time point was 
chosen because the rate of change in OD600 decreased after two hours. The results showed that increasing gel concentration from $0 \%$ to $0.75 \%$ (weight/volume) caused a

1231.8 fold reduction in final bacterial density (Fig. 2A). This suggests that DH5 $\alpha$ cells grew

124 slower with increasingly dense hydrogel, resulting in reduced growth.

Figure 2
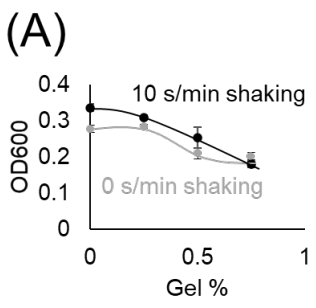

(D)

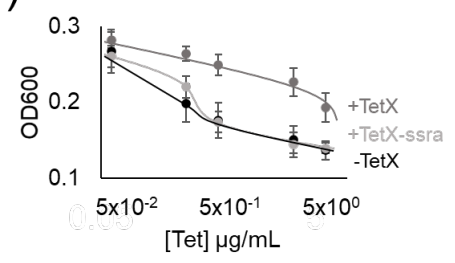

(C)
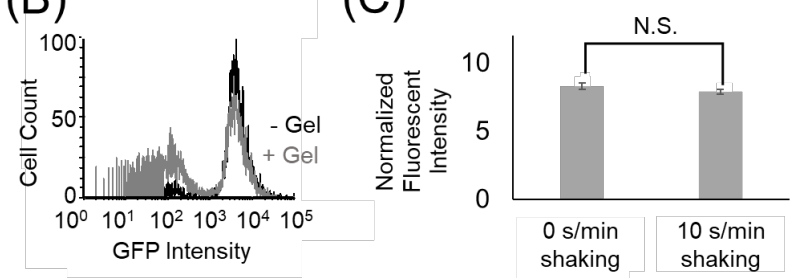

(E)

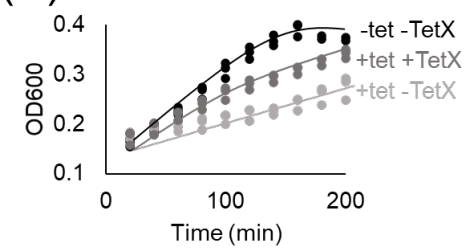

Figure 2. Hydrogel encapsulation imparts gene expression heterogeneity

(A) Cell density in different gel concentration environments at $2 \mathrm{hr} .0 \%, 0.25 \%, 0.5 \%$ and $0.75 \%$ gel density with $10 \mathrm{~s} / \mathrm{min}$ shaking (black line) or with no shaking (grey line). Increased hydrogel concentration reduces bacterial growth. Points represent mean values, and error bars represent standard error of the mean. Mean and SEM are calculated using six biological replicates.

(B) Fluorescence intensity distribution of cells expressing TetX-GFP fusion protein in media containing $0 \%$ (black line) and $0.5 \%$ low-melting-temperature agarose (grey line). Results demonstrate that hydrogel-encapsulation limits the expression of TetX-GFP in a subset of bacteria. One of two replicates shown for each condition, refer to Supp. Fig. 4 for both replicates.

(C) Fluorescence intensity of TetX-GFP normalized by OD600 of cells after two hours of growth with either $0 \mathrm{~s} / \mathrm{min}$ or $10 \mathrm{~s} / \mathrm{min}$ shaking. Mean expression levels are not significantly different. Two-tailed, equal variance t-test returns $p$-value $=0.189$. N.S. stands for 'not significant'. Bar height represents mean values, and error bars represent standard error of the mean. Mean and SEM are calculated using eight biological replicates.

(D) Cell density of resistant and non-resistant $\mathrm{DH} 5 \alpha$ cells under different concentrations of tetracycline. +TetX-ssra (light grey line), +TetX (grey line) and -TetX (black line). Results show that TetX rescues bacterial growth from tetracycline repression. Points represent mean values, and error bars represent standard error of the mean. Mean and SEM are calculated using six biological replicates.

(E) Cell density of resistant bacteria (TetX) in different tetracycline (tet) over time. $0.625 \mu \mathrm{g} / \mathrm{mL}$ tetracycline on DH5 $\alpha$ cells without TetX (light grey line), $0.625 \mu \mathrm{g} / \mathrm{mL}$ tetracycline on $\mathrm{DH} 5 \alpha$ cells without TetX (grey line) and $0 \mu \mathrm{g} / \mathrm{mL}$ tetracycline on $\mathrm{DH} 5 \alpha$ cells without TetX (black line). Results show that tetracycline inhibition of cellular growth is reflected by decreased rates of growth over time, and rescue by TetX is reflected by increased rates of growth over time. Points represent individual replicates. Six replicates per condition at each time point.

To establish the effect of exogenous hydrogel on gene expression modules,

126 DH5 $\alpha$ cells constitutively expressing GFP (Methods- Section B) were grown in media

127 with $0.5 \%$ gel $(\mathrm{w} / \mathrm{v})$ or $0 \%$ gel. Single-cell expression data from $\mathrm{DH} 5 \alpha$ cells grown in the 
presence of hydrogel showed that a secondary population of low-fluorescence cells appeared (Fig. 2B, Supp Fig. 4, Methods- Section E). This bimodal expression distribution suggests that hydrogel encapsulation imparts heterogeneous growth conditions that stochastically limit gene module expression levels, splitting the cellular population into "on" and "off" states. Taken together, hydrogel-mediated reductions in growth and gene expression are consistent with previous work on microbes in heterogeneous environments (Alvarez et al., 2009; Pabst et al., 2016; Wessel et al., 2013). Notably, changes in periodic perturbation did not significantly alter populationlevel fluorescence measurements (Fig. 2C, Supp Fig. 5). This observation could be explained by periodic perturbation inducing offsetting changes in gene expression rate and protein dilution rate.

To test the effect of protein expression on bacterial growth during encapsulation, a genetic module that constitutively expresses TetX was constructed (Ghosh et al., 2015) (Methods- Section A). We validated the efficacy of this TetX module by growing resistant and non-resistant bacteria at a series of tetracycline concentrations, demonstrating that TetX expression allows cells to grow to a 1.5-fold greater final density in the presence of $5 \mu \mathrm{g} / \mathrm{mL}$ tetracycline (Fig. 2D). This change in density is also reflected over time (Fig. 2E). To confirm that the protein product of the module (i.e. TetX) was imparting antibiotic resistance, an unstable version of TetX was constructed by fusing a degradation tag to TetX (Gottesman et al., 1998). The unstable protein either imparted very weak tetracycline resistance or no detectable tetracycline resistance (Fig. 2D). These results establish that Tet $X$ expression, rather than nonspecific protection from the TetX module, allows cells to effectively resist tetracycline (Fig. 2D-E).

\section{Bacteria exhibit emergent growth in a 3D fluctuating hydrogel}

To examine the population-level consequences of functional protein expression

155 for bacteria inside a periodically-perturbed hydrogel, the growth of $\mathrm{DH} 5 \alpha$ cells 156 constitutively expressing TetX was measured over time (Fig. 3A, Methods- Section F). $1570,0.3125$, or $0.625 \mu \mathrm{g} / \mathrm{mL}$ tetracycline was supplemented in the media. To quantify 
relative growth, the final density of cells expressing the TetX module was normalized by initial density, and the growth of naïve cells cultured under the same conditions. Under all growth conditions, larger concentrations of tetracycline increased the relative growth of TetX-expressing cells. The significance of increases or decreases in relative growth between cells at different concentrations of hydrogel were assayed using a Bonferroni

Figure 3

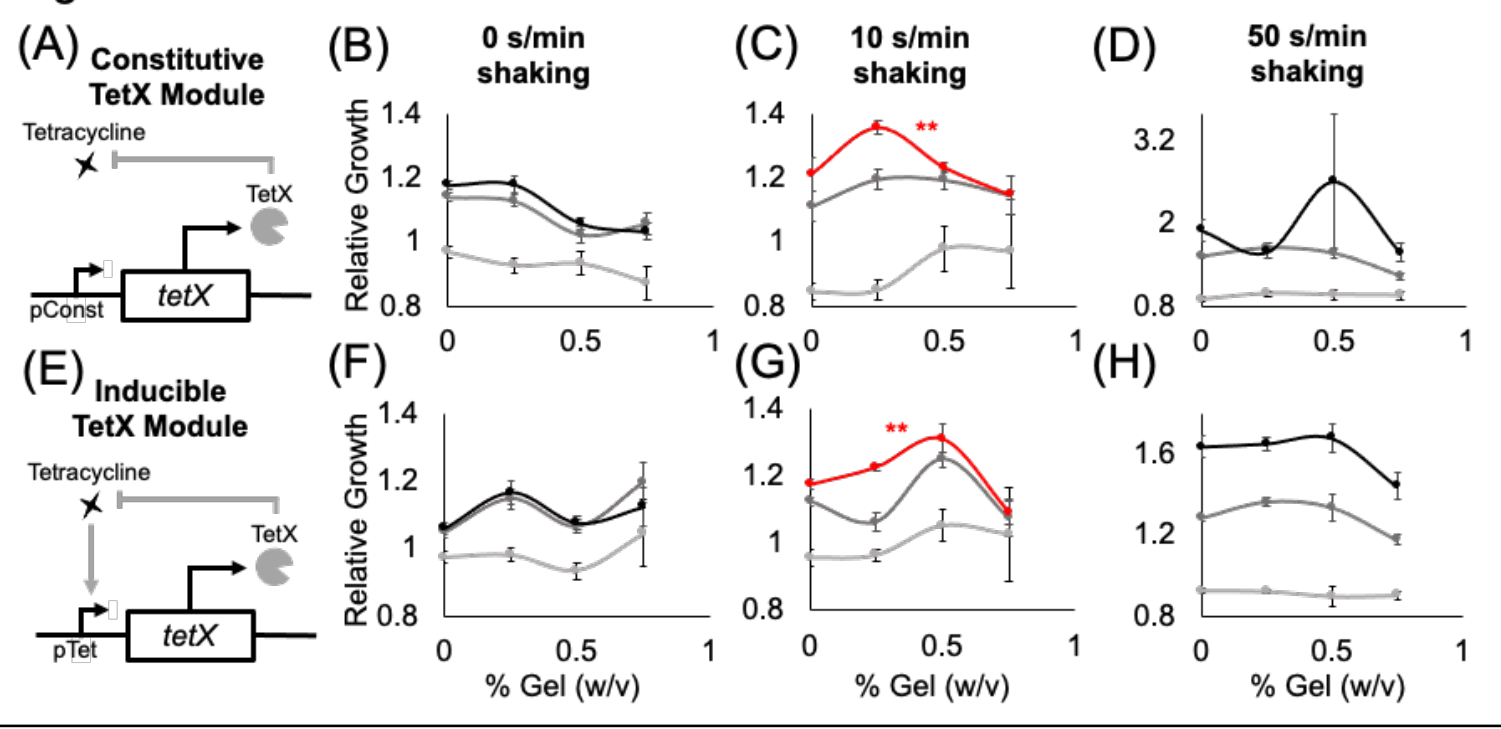

Figure 3. A minimal antibiotic-resistance module enables frequency-dependent response of bacterial growth in hydrogel.

(A) Abstract depiction of tetracycline degradation by constitutive TetX expression module. (B-D) Relative growth of $E$. coli cells constitutively expressing TetX without shaking (B), with $10 \mathrm{~s}$ shaking (C), with 50 s shaking (D). Light grey is $0 \mu \mathrm{g} / \mathrm{mL}$ tetracycline, dark grey is $0.3125 \mu \mathrm{g} / \mathrm{mL}$ tetracycline, black is $0.625 \mu \mathrm{g} / \mathrm{mL}$ tetracycline. The red line represents a statistically significant biphasic curve as judged by a Bonferroni multiple comparisons test (treated with $0.625 \mu \mathrm{g} / \mathrm{mL}$ tetracycline). Points represent mean values, and error bars represent standard error of the mean. (B) and (C) Mean and SEM are calculated using six biological replicates. (D) Mean and SEM are calculated using four biological replicates.

(E) Abstract depiction of feedback between bacterial growth and inducible TetX expression module when feeding tetracycline.

$(\mathrm{F}-\mathrm{H})$ Relative growth of $E$. coli cells inducibly expressing TetX without shaking (F), with 10 s shaking $(\mathrm{G})$, with 50 s shaking $(\mathrm{H})$. Light grey is $0 \mu \mathrm{g} / \mathrm{mL}$ tetracycline, dark grey is $0.3125 \mu \mathrm{g} / \mathrm{mL}$ tetracycline, black is $0.625 \mu \mathrm{g} / \mathrm{mL}$ tetracycline. The red line represents a statistically significant biphasic curve as judged by a Bonferroni multiple comparisons test (treated with $0.625 \mu \mathrm{g} / \mathrm{mL}$ tetracycline). Points represent mean values, and error bars represent standard error of the mean. Mean and SEM are calculated using six biological replicates.

At $0 \mathrm{~s} / \mathrm{min}$ shaking and 0.625 or $0.3125 \mu \mathrm{g} / \mathrm{mL}$ tetracycline, the relative growth of 
columns, Supp. Fig. 7A mid \& bottom rows). With $0 \mathrm{~s} / \mathrm{min}$ shaking and $0 \mu \mathrm{g} / \mathrm{mL}$ tetracycline, relative growth did not significantly change as gel concentration increased (Fig. 3B light gray, Supp. Fig. 6A $1^{\text {st }}$ column, Supp. Fig. 7A top row). At $10 \mathrm{~s} / \mathrm{min}$ shaking and $0.625 \mu \mathrm{g} / \mathrm{mL}$ tetracycline, cells with the TetX module exhibited a significant biphasic change in relative growth as gel concentration increased (Fig. $3 \mathrm{C}$ red, Supp. Fig. 6B $3^{\text {rd }}$ column, Supp. Fig. 7B bottom row). With $10 \mathrm{~s} / \mathrm{min}$ shaking and 0 or 0.3125 $\mu \mathrm{g} / \mathrm{mL}$ tetracycline, the change in relative growth was visually biphasic, but had no significant trend (Fig. 3C, dark and light grey, Supp. Fig. 6B $1^{\text {st }} \& 2^{\text {nd }}$ columns, Supp. Fig. 7B top and mid row). These results suggest that periodic perturbation interacts with TetX expression to allow bacteria to enhance growth in a manner sensitive to hydrogel concentration.

To distinguish whether biphasic growth was a general response to the presence of shaking or a unique response to a specific frequency of shaking, we gathered additional data from cells shaken at $50 \mathrm{~s} / \mathrm{min}$. At $50 \mathrm{~s} / \mathrm{min}$ shaking and $0.3125 \mu \mathrm{g} / \mathrm{mL}$ tetracycline, relative growth decreased as hydrogel concentration increased (Fig. 3D, dark grey, Supp. Fig. 6C $2^{\text {nd }}$ column, Supp. Fig. $7 \mathrm{C}$ mid row). At $50 \mathrm{~s} / \mathrm{min}$ shaking and 0 or $0.625 \mu \mathrm{g} / \mathrm{mL}$ tetracycline, relative cell growth did not significantly change (Fig. 3D black \& light grey, Supp. Fig. 6C $1^{\text {st }} \& 3^{\text {rd }}$ columns, Supp. Fig. 7C top \& bottom rows). These results suggest the biphasic response of TetX-expressing cells grown with 10 $\mathrm{s} / \mathrm{min}$ shaking and $0.625 \mu \mathrm{g} / \mathrm{mL}$ tetracycline (Fig. $3 \mathrm{C}$ red) is based on the frequency of periodic perturbation rather than the presence of perturbation.

To test whether the frequency-specific biphasic bacterial growth curve was mediated by the expression dynamics of the constitutive TetX module, we built and tested an inducible TetX module under the same shaking conditions and hydrogel concentrations. Inducible TetX expression was achieved by transforming the constitutive TetX module into a cell line (DH5 $\alpha$ pro) that regulated module expression via genomically integrated tetR (Fig. 3E, Supp. Fig. 8, Methods- Section B). At $0 \mathrm{~s} / \mathrm{min}$ shaking, no tetracycline concentrations produced a significant change in relative growth as hydrogel concentration rose (Fig. 3F, Supp. Fig. 6D, Supp. Fig. 9A). At $10 \mathrm{~s} / \mathrm{min}$ shaking and $0.625 \mu \mathrm{g} / \mathrm{mL}$ tetracycline, the inducible TetX module displayed a significant 
197 biphasic relationship between relative growth and hydrogel concentration (Fig. 3G red,

198 Supp. Fig. 6E $3^{\text {rd }}$ column, Supp. Fig. 9B bottom row). This biphasic growth curve of the 199 inducible module was shifted to the right of the constitutive module growth curve,

200 suggesting that the activation kinetics of TetX production affect population-level

201 integration of spatial and temporal information. At $10 \mathrm{~s} / \mathrm{min}$ shaking and 0 or 0.3125

$202 \mu \mathrm{g} / \mathrm{mL}$ tetracycline, bacteria inducibly expressing TetX experienced no significant

203 change in relative growth as hydrogel concentration rose (Fig. 3G light and dark grey,

204 Supp. Fig. 9B top \& middle rows). At $50 \mathrm{~s} / \mathrm{min}$ shaking and 0, 0.3125, or $0.625 \mu \mathrm{g} / \mathrm{mL}$

205 tetracycline, the inducible TetX module similarly displayed no significant change in

206 relative growth as hydrogel concentration increased (Fig. 3H, Supp. Fig. 6D, Supp Fig.

207 9C). Taken together, the results from the constitutive and inducible TetX modules (Fig.

208 3, Supp. Fig. 6, Supp. Fig. 9) demonstrate that the genetic regulation of an antibiotic

209 resistance gene tunes the hydrogel sensitivity of bacterial growth under tetracycline

210 exposure.

211 A stochastic resonance model can explain the emergent growth dynamics of bacteria

212 After observing counterintuitive, frequency-sensitive bacterial growth, we sought

213 to build a model to explain this phenomenon. Since protein expression level is similar

214 between different shaking frequencies (Fig. 2C), differences in mean expression level

Figure 4
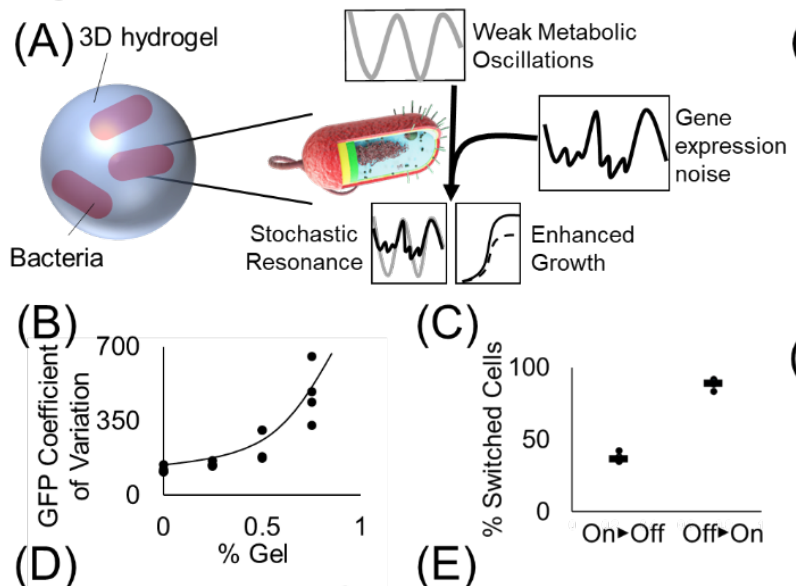

(D)

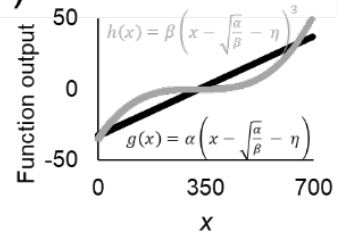

(C)

(E)
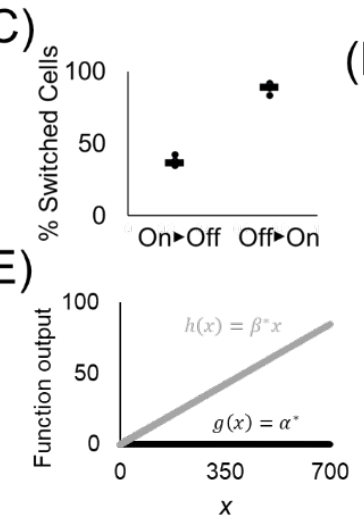

$(\mathrm{F})$

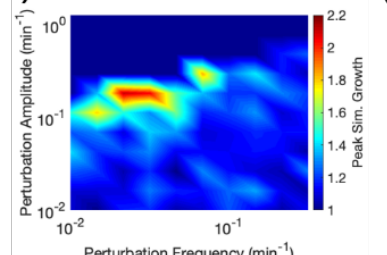

$(\mathrm{H})$

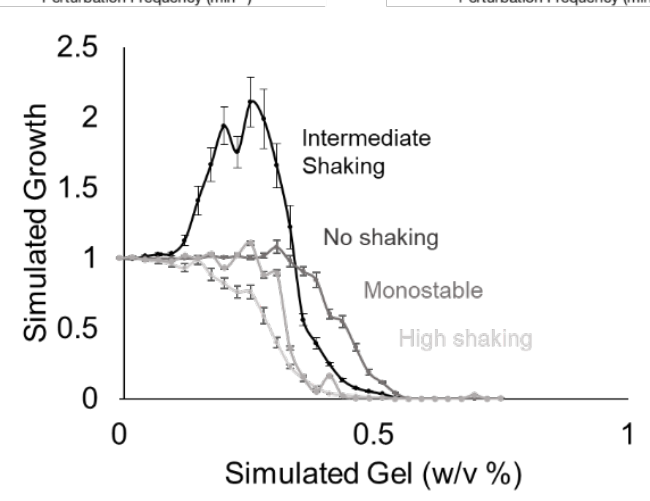

(G)

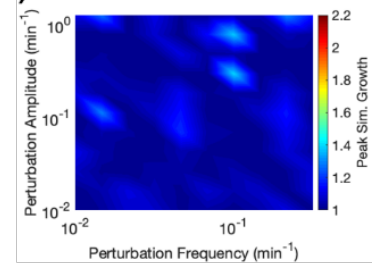


Figure 4. Simulated stochastic resonance generates biphasic, frequency-dependent bacterial growth

(A) Cartoon of the proposed model of bacterial stochastic resonance. At a specific frequency shaking, weak metabolic oscillations resonate with stochastic fluctuations in TetX production, boosting growth in tetracycline-treated bacteria.

(B) Coefficient of variation of bacterial GFP expression in hydrogel. Graph shows that increased hydrogel concentration increases the variability of genetic module expression. Points represent individual replicates, line highlights trend. Four replicates per condition. Lines represent the median proportion of switched bacteria.

(C) Quantification of 'on' and 'off' cellular populations that grew from cells sorted in the opposite state. Results suggest that gene expression can switch between 'on' and 'off' expression modes. Points represent individual replicates. Four replicates per condition.

(D) Output of the positive $(h(x)$, grey line) and negative function $(g(x)$, black line) that represent a system with two stable fixed points.

(E) Output of the positive $(h(x)$, grey line) and negative function $(g(x)$, black line) that represent a system with one stable fixed point.

(F) Heat plot showing the simulated growth of bacteria at different frequencies and amplitudes of periodic perturbation. Color indicates the maximum growth of bacteria over a range of simulated hydrogel concentrations normalized by the growth of bacteria at the lowest gel concentration. Red areas show regions where stochastic resonance is causing biphasic changes in bacterial growth with respect to gel concentration. Surface plot values represent mean values. The mean is calculated using two hundred simulation replicates.

(G) Heat plot showing the simulated growth of bacteria that cannot achieve bistable growth at different frequencies and amplitudes of periodic perturbation. Color indicates the maximum growth of bacteria over a range of simulated hydrogel concentrations normalized by the growth of bacteria at the lowest gel concentration. The results indicate that stochastic resonance does not occur under these conditions. Surface plot values represent mean values. The mean is calculated using two hundred simulation replicates.

(H) Simulated bacterial growth in different hydrogel concentrations. Black line represents simulation with the amplitude, frequency, and bistable growth required for stochastic resonance. Black line shows growth under stochastic resonance conditions causes a biphasic relationship between the total growth in the system and the simulated gel concentration, similar to the $10 \mathrm{~s} / \mathrm{min}$ shaking conditions in Fig. 3. Dark grey represents simulation with a periodic amplitude of zero, similar to the $0 \mathrm{~s} / \mathrm{min}$ shaking conditions. Intermediate grey represents a simulation with a monostable system. Light grey represents a simulation with a reduction in the frequency of periodic perturbation. Results demonstrate that changing periodic forcing amplitude, frequency, or the underlying bistability of growth eliminates biphasic growth with respect to simulated gel concentration. Points represent mean values, and error bars represent standard error of the mean. Mean and SEM are calculated using two hundred simulation replicates.

are unlikely to drive biphasic growth with respect to hydrogel concentration.

216 Alternatively, frequency-specific, biphasic responses have been previously associated

217 with stochastic resonance in biological systems (McDonnell and Abbott, 2009; Paulsson

218 et al., 2000; Zeng et al., 2009). For stochastic resonance theory to apply, there must be

219 a weak oscillatory force and a source of biochemical noise (Fig. 4A).

The growth rate characterization experiments for Perceval show that there is an

221 approximately $18 \%$ difference between the ATP/ADP ratio of cells with a significantly

222 different growth rate (Fig. 1B). When cells are periodically perturbed, the maximum 
223 versus minimum A490/A405 measurements show the peak versus the trough of

224 ATP/ADP oscillations are approximately 6\% different (Fig. 1C-D). These results show

225 that that shaking can exert an oscillatory force on bacterial metabolism that is not

226 necessarily strong enough to be associated with a significant change in growth rate.

227 This, in turn, suggests that periodic shaking exerts a weak oscillatory influence on 228 growth.

$229 \quad$ Hydrogels have been hypothesized to generate extrinsic noise in encapsulated 230 bacteria. Because real-time fluctuation of protein levels in single planktonic cells under 231 shaking conditions cannot be measured, we used flow cytometry to infer the effect of 232 hydrogel encapsulation on gene expression noise. Bacteria encapsulated in hydrogel 233 experience an increase in the coefficient of variation of fluorescent reporter expression

234 (Fig. 4B) caused by the splitting of the population between 'on' and 'off' expression 235 modes (Fig. 2B). We tested the ability of cells grown in hydrogel to switch between 'on' 236 and 'off' states by sorting the two populations, growing them independently, then 237 quantifying changes in the proportion of 'on' vs. 'off' cells (Methods- Section H). A 238 mixture of 'on' and 'off' states were observed in cultures grown from cells started from 239 exclusively one state. These results suggest that cells dynamically switch between 'on' 240 and 'off' states. These results build off prior work showing that synthetic materials can 241 increase bacterial heterogeneity (Alvarez et al., 2009; Pabst et al., 2016; Wessel et al., 242 2013), demonstrating that cells grown in hydrogel exhibit dynamic fluctuations of gene 243 expression (Fig. 4C). Based on these experiments, we assert that hydrogel causes 244 dynamic fluctuations in TetX expression, imparting noise on the growth rate of 245 encapsulated cells.

246 After establishing the biological criteria for stochastic resonance in synthetically 247 encapsulated bacteria, we modified a classical stochastic differential equation (Eq. 1) 248 (Gammaitoni et al., 1998) to describe the effect of stochastic resonance on bacterial 249 growth (Methods- Section I).

$250 \quad \frac{d \mu}{d t}=\alpha\left(x-\sqrt{\frac{\alpha}{\beta}}-\eta\right)-\beta\left(x-\sqrt{\frac{\alpha}{\beta}}-\eta\right)^{3}+\Lambda x \cos (\Omega t)+\sigma \xi(t)$

251 Where $\mu$ is the growth rate, $x$ is the concentration of cells normalized by the maximum 
252 concentration of cells, $t$ is time, $\alpha$ and $\beta$ represent the growth of bacteria expressing the

253 TetX module under tetracycline treatment $\left(\mathrm{min}^{-1}\right), \eta$ represents the basal bacterial

254 concentration normalized by the maximum concentration of cells, $\Lambda$ is the amplitude of a

255 weak periodic effect on growth $\left(\mathrm{min}^{-1}\right), \Omega$ is the period of a weak periodic effect on

256 growth $\left(\min ^{-1}\right)$, and $\sigma$ is the noise magnitude $\left(\min ^{-1}\right)$.

The bacterial growth term represented by $\alpha, \beta$, and $\eta$ was chosen to represent bistable growth caused by the feedback between importation, degradation, and translation-inhibition activity of antibiotics as described by Deris et. al. (Deris et al., 2013). To verify the bistability of the equation, we plotted the output of the positive and negative function terms to identify the stable and unstable fixed points (Fig 4D). We also implemented a simpler equation (Eq. 2) to represent non-bistable (i.e. monostable) growth of bacteria in the absence of tetracycline.

$\frac{d \mu}{d t}=\alpha^{*}-\beta^{*} x+\Lambda x \cos (\Omega t)+\sigma \xi(t)$

Where $\mu$ is the growth rate, $x$ is the concentration of cells normalized by the maximum concentration of cells, $t$ is time, $\alpha^{*}$ and $\beta^{*}$ represent the growth of bacteria in the absence of tetracycline $\left(\mathrm{min}^{-1}\right), \Lambda$ is the amplitude of a weak periodic effect on growth $\left(\min ^{-1}\right), \Omega$ is the period of a weak periodic effect on growth $\left(\mathrm{min}^{-1}\right)$, and $\sigma$ is the noise magnitude $\left(\mathrm{min}^{-1}\right)$. A parameter set for Eq. 2 was empirically derived to quantitatively match the integral of the growth rates from Eq. 1. The stability diagram of Eq. 2 was plotted under the chosen parameters, demonstrating that it is not bistable (Fig. 4E).

Next, Eq. 1 and 2 were used to simulate bacterial growth with weak periodic

273 perturbation of growth rates. To capture the effect of hydrogel encapsulation on

274 bacterial growth rate, noise was applied to the system such that increased the

275 heterogeneity of growth rates while also decreasing the overall growth rate. Simulations

276 showed that at a narrow range of amplitude and frequency, the relative bacterial growth

277 rate over a range of simulated gel concentrations was enhanced (Fig. 4F). Simulated

278 bacterial growth without bistability did not experience frequency and noise specific

279 enhancements (Fig. 4G). 
The simulated growth of bacteria experiencing frequency-dependent enhancement was plotted against the range of simulated gel concentrations (Fig. $\mathbf{4 H}$ black line). Under conditions where relative growth enhancement was reported, simulated growth was found to be biphasic as simulated hydrogel concentrations increased (Fig. 4H black line). Removing the weak periodic force (Fig. 4H dark grey), changing the underlying bistability (Fig. 4H mid grey), or altering the periodic forcing frequency (Fig. 4H light grey) eliminated the biphasic growth with respect to simulated hydrogel concentration. These simulations demonstrate that stochastic resonance is sufficient to explain the biphasic, frequency-dependent bacterial growth curves that we experimentally observed in tetracycline-resistant bacteria.

\section{Discussion}

Encapsulation of designer bacteria into hydrogels has birthed a new class of smart devices that can internally coordinate decision-making. These engineered living materials require novel strategies to maintain the potency of their biological components under restrictive metabolic conditions. To address this need, the effects of internal genetic machinery and external kinetic perturbation were systematically investigated in bacteria encapsulated in a three-dimensional hydrogel. An antibiotic resistance module was shown to optimize bacterial growth under restrictive encapsulation conditions based on the frequency of a rapid periodic disturbance. A simple biophysical model (Eq.

1) of stochastic resonance was proposed to explain this information processing phenomenon that appears to coordinate bacterial growth in three-dimensional hydrogels.

The model developed in this study provides a framework to maximize the efficiency of encapsulated bacterial growth by coordinating the timing of intra- and extracellular fluctuations. This model could be used as a guide for bacteria-seeded materials with gene expression noise tuned to resonate with a natural periodic process like peristalsis. Conversely, this model could also be used to determine which physical properties of an encapsulating substrate would be optimal to boost pre-existing cellular processes. 
This study invites several lines of future inquiry. New strains and encapsulated substrates may be tested to create more systematic guidelines for the application of stochastic resonance to engineered living materials. To explain why constitutive TetX-

314 producing cells have a distinct optimal hydrogel concentration from inducible TetX-

315 producing cells, additional genetic circuits could be used to explore which regulatory

316 features mediate the integration of spatial and temporal information. Given the

317 frequency sensitivity of stochastic resonance, full confirmation of the model proposed in

318 this study will require real-time tracking and measurement of single-cell gene

319 expression in a semisolid environment. Further high-level evidence for stochastic

320 resonance could also be collected by identifying a periodic shaking frequency that shifts

321 the biphasic growth curve rather than eliminating it.

\section{Methods}

\section{Contact for reagent and resource sharing}

325 Further information and requests for resources and reagents should be directed to and 326 will be fulfilled by the Lead Contact, Cheemeng Tan (cmtan@ucdavis.edu).

\section{A. Media preparation and handling}

329 All media in this study was prepared from granulated Miller's Luria Broth from Research

330 Products International. Seaplaque low-melting-temperature agarose (VWR) was

331 incorporated into the media before autoclaving. Media was autoclaved for 30 minutes at

$332121^{\circ} \mathrm{C}$. Sterilization and pressure release took one hour, after which media was cooled 333 on the bench for 10 minutes before being placed in a $37^{\circ} \mathrm{C}$ incubator and being shaken

334 at $200 \mathrm{rpm}$. During the course of experiments where cells were grown with low-melting335 temperature-agarose, media was placed on a hotplate kept at $40^{\circ} \mathrm{C}$ while it was in use, 336 then returned to the shaker when it was not in use. Media was made fresh the day 337 before every experiment.

338 Consistent sterilization time and continuous heating of media containing low-melting 339 temperature agarose were found to be critical for the reproducibility of all results.

340 Concentrations of low-melting-temperature agarose above .75\% increased the 341 variability of the individual replicates such that reproducible results were not possible 
342 and were thus excluded from this study.

B. Plasmid construction and expression control

All plasmids used in this study were generated by Gibson cloning. The coding sequence for TetX was ordered from Genscript. The TetX sequence, the GFP sequence, all promoters, ribosome binding sites, and degradation tags were amplified by PCR with Q5 polymerase (NEB), gel extracted (Qiagen), and quantified using a Nanodrop (Thermo Fisher). For the constitutive and inducible circuits, TetX and GFP sequences were assembled with $\mathrm{P}_{\mathrm{Tet}}, \mathrm{RBS}$, and ssra tags as necessary in the $\mathrm{pZ}$ backbone that had been digested with EcoRI and BamHI with buffer 3.1 (all NEB) at $37^{\circ} \mathrm{C}$ in a static incubator overnight. For the inducible circuits, expression was controlled by a gnomically integrated copy of TetR in the line DH5 $\alpha$ pro. DNA fragments were assembled via the Gibson Assembly Master Mix (NEB) for 1 hour at $50^{\circ} \mathrm{C}$ following the DNA molar ratios suggested by the company's protocol. Successful transformations were assayed by digestion with $\mathrm{Ncol}, \mathrm{BamHI}$, and buffer 3.1 and confirmed by Sanger sequencing. The plasmid for the luminescence tests was pCS-PEsaR-Plux, a gift from Cynthia Collins (Addgene plasmid \#47655). E. coli DH5a/pRsetB-his7-Perceval was a gift from Gary Yellen's lab (Addgene plasmid \#20336). Plasmid pRsetB-his7-Perceval was isolated and transferred into E. coli DH5a/pSC-Ptet-T7 which expresses T7 polymerase constitutively.

A single colony of E. coli DH5a/pSC-P tet-T7/pRsetB-his7-Perceval was picked and inoculated into LB with $50 \mu \mathrm{g} / \mathrm{mL}$ kanamycin and $100 \mu \mathrm{g} / \mathrm{mL}$ carbenicillin overnight at $37^{\circ} \mathrm{C}$ at $120^{\circ}$ tilt from horizontal with 200 rpm shaking.

367 For quantification of ATP/ADP in M9 media, the overnight culture was subcultured into M9 with $0.05 \%, 0.1 \%, 0.2 \%$ and $0.4 \%$ glucose with the corresponding antibiotic with an initial OD $600 \mathrm{~nm}$ of 0.05 . Next, $200 \mu \mathrm{L}$ cell culture was put inside 96 well black plate with a clear flat bottom (Corning, ref \#3631) combined with a clear lid. ATP/ADP ratios were measured by taking three rounds of reading at excitation $495 \mathrm{~nm} /$ emission $530 \mathrm{~nm}$, and 
$372405 \mathrm{~nm} / \mathrm{emission} 530 \mathrm{~nm}$ by plate reader in a 2 mins cycle with $10 \mathrm{~s}$ orbital shaking

373 (amplitude 3). T-tests were performed in Microsoft Excel using two tails and unequal

374 variance.

375 For quantification of ATP/ADP in LB medium, the overnight culture was subcultured into 376 fresh LB at $37^{\circ} \mathrm{C}$ at $120^{\circ}$ tilt from horizontal with $200 \mathrm{rpm}$ shaking with initial $\mathrm{OD}_{600}$ $377 \mathrm{~nm}=0.05, \mathrm{~A} 490$ and A405 values of $E$. coli DH5a/pSC-P tet-T7/pRsetB-his7-Perceval in 378 LB medium with glucose were recorded using plate reader. ATP/ADP ratios were measured by taking three rounds of reading at excitation $495 \mathrm{~nm} / \mathrm{emission} 530 \mathrm{~nm}$, and $405 \mathrm{~nm} / \mathrm{emission} 530 \mathrm{~nm}$ by plate reader in a 2 mins cycle with $10 \mathrm{~s}$ orbital shaking (amplitude 3). Subsequent readings were analyzed by Fourier transform in MATLAB using the fft function. Significance between differences in fluctuation amplitude was measured by a two-tailed, unequal variance t-test in Microsoft Excel.

All luminescence results were generated using a plate reader (Tecan M1000pro). Cells were grown overnight at $37^{\circ} \mathrm{C}$ at $120^{\circ}$ tilt from horizontal with $200 \mathrm{rpm}$ shaking in LB with antibiotics from a single colony, then diluted to a constant OD600 value of 0.5 . All cell lines were incubated on ice for 30 minutes. Cell cultures standardized in this way were further diluted 1:100 into LB media with the appropriate antibiotics and the appropriate concentration of low-melting-temperature agarose as indicated in the data. DH5a cells were grown in media without antibiotics, the luminescent cell line was grown with $30 \mu \mathrm{g} / \mathrm{mL}$ kanamycin. These cells were incubated for 1.5 hours at $37^{\circ} \mathrm{C}$ at $120^{\circ}$ tilt from horizontal with $200 \mathrm{rpm}$ shaking until they reached the beginning of exponential growth, at which point they were distributed into a 96-well plate, covered with a lid, and put into the plate reader at $37^{\circ} \mathrm{C}$. Total well volume was $200 \mu \mathrm{L}$. The plate reader subjected the cells to 10 seconds of orbital shaking (amplitude 3 ), iterated through three rounds of reading luminescence then OD600, then restarted the cycle. Each cycle took two minutes. Readings were taken for at least six hours. Comparisons between shaking and non-shaking conditions were made by picking times points where the OD600 readings of the shaking and non-shaking cultures were equal to 0.25 . 


\section{E. Flow cytometry for cells expressing GFP}

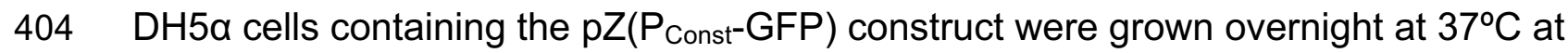
$405120^{\circ}$ tilt from horizontal with $200 \mathrm{rpm}$ shaking in LB with $30 \mu \mathrm{g} / \mathrm{mL}$ kanamycin from a 406 single colony, then diluted to a constant OD600 value of 0.5 . Cells were incubated on 407 ice for 30 minutes, then further diluted 1:100 into LB media with $30 \mu \mathrm{g} / \mathrm{mL}$ kanamycin 408 and the appropriate concentration of low-melting-temperature agarose as indicated in 409 the data. These cells were incubated for 1.5 hours at $37^{\circ} \mathrm{C}$ at $120^{\circ}$ tilt from horizontal 410 with 200 rpm shaking until they reached the beginning of exponential growth, at which 411 point they were distributed into a 96-well plate, covered with a lid, and put into the plate 412 reader at $37^{\circ} \mathrm{C}$. Total well volume was $200 \mu \mathrm{L}$. OD600 and GFP fluorescent intensity of 413 each well was monitored in the plate reader with measurements taken every 10 414 minutes, with 10 seconds of orbital shaking (amplitude 3) every minute. Cells were 415 grown in the plate for 2 hours before being diluted 1:100 into PBS with .4\% paraformaldehyde. Fixed cells were run on a Novocyte Flow Cytometer (ACEA

417 Biosciences). At least nineteen thousand events were collected for each sample, and 418 data from each sample was analyzed using FCS Express version 4.0 (De Novo

419 Software, Los Angeles, CA).

\section{F. Characterization of biochemical circuits and relative growth}

422 All growth results were generated using a plate reader (Tecan M1000pro). Cells were 423 grown overnight at $37^{\circ} \mathrm{C}$ at $120^{\circ}$ tilt from horizontal with $200 \mathrm{rpm}$ shaking in LB with

424 antibiotics from a single colony, then diluted to a constant OD600 value of 0.5. All cell

425 lines were incubated on ice for 30 minutes to ensure uniform growth between different 426 cell lines. Cell cultures standardized in this way were further diluted 1:100 into LB media 427 with the appropriate antibiotics and the appropriate concentration of low-melting428 temperature agarose as indicated in the data. The non-resistant cell lines, $\mathrm{DH} 5 \alpha$ and 429 DH5apro, were grown in media without antibiotics, both constitutive lines and the 430 inducible lines were grown with $30 \mu \mathrm{g} / \mathrm{mL}$ kanamycin, and the oscillator TetX lines were 431 grown with $30 \mu \mathrm{g} / \mathrm{mL}$ kanamycin and $50 \mu \mathrm{g} / \mathrm{mL}$ carbenicillin. These cells were incubated 432 for 1.5 hours at $37^{\circ} \mathrm{C}$ at $120^{\circ}$ tilt from horizontal with $200 \mathrm{rpm}$ shaking until they reached 
433 the beginning of exponential growth, at which point they were distributed into a 96-well

434 plate, covered with a lid, and put into the plate reader at $37^{\circ} \mathrm{C}$. Total well volume was

$435200 \mu \mathrm{L}$. OD600 and GFP fluorescent intensity of each well was monitored in the plate

436 reader with measurements taken every 10 minutes. Without shaking, the plate was left

437 undisturbed between measurements. Under intermediate shaking conditions, the plate

438 experienced 10 seconds of orbital shaking (amplitude 3) every minute. Under high

439 shaking conditions, the plate experienced 50 seconds of orbital shaking (amplitude 3)

440 every minute. Cells were allowed to grow for 2 hours before $50 \mu \mathrm{L}$ of culture per well

441 was transferred into a new 96-well plate with $150 \mu \mathrm{L}$ of media already in the plate along

442 with indicated concentrations of tetracycline. Unless otherwise noted, all results were

443 taken from cells after 120 minutes of growth in the second plate. Unless otherwise

444 noted, all growth results were replicated 6 times: three sets of duplicated samples were

445 collected on distinct days. No blinding or randomization was performed. A sample size

446 of six was selected according to standard synthetic biology practices of sample

447 replication inside a plate reader for significance testing, but these tests have no

448 established effect size.

\section{G. Significance testing on relative growth curves}

452 Since we are trying to determine the significance of differences between many points in

453 a gene expression transfer function, we chose to use a multiple comparisons test

454 instead of a normal t-test. For experimental data, each relative growth curve (plotted

455 with respect to noise intensity) was subjected to a Bonferroni multiple comparisons test,

456 which performs significance testing between each point within the transfer function,

457 correcting for false positives by adjusting the $p$-value of the test by dividing it by the

458 number of comparisons being made. With this technique, we established confidence

459 intervals between the values in each relative growth curve using a base $p$-value of 0.05.

460 If the curve experienced a significant increase in cell growth followed by a significant

461 decrease, that curve was classified as biphasic. 


\section{H. Measurement of stochastic switching expression in noisy environments}

465 In brief, we followed the distribution of cells that started unimodally "on" and "off", then used flow cytometry to characterize the bimodal populations of cells that were present at the end of the experiment and counted the proportion of the final population that had switched states. This experiment was broken into two phases to account for the competitive advantage of "on" cells under tetracycline treatment. The first phase starts at a high density of constitutively active cells before measuring their fluorescent gene expression while growing in $0.5 \%$ low-melting-temperature agarose. The second phase starts with a low density of cells sorted to be genetically inactive before measuring their fluorescent gene expression while growing in $0.5 \%$ low-melting-temperature agarose.

474 To measure the switching of cells from an "on" state to an "off" state, DH5a cells

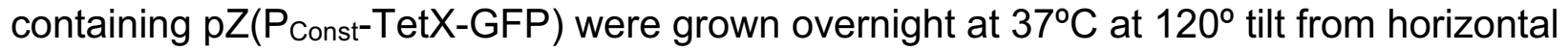
with $200 \mathrm{rpm}$ shaking in LB with $30 \mu \mathrm{g} / \mathrm{mL}$ kanamycin from a single colony, then diluted to a constant OD600 value of 0.5. Cells were incubated on ice for 30 minutes, then further diluted 1:100 into LB media with $30 \mu \mathrm{g} / \mathrm{mL}$ kanamycin and 0.5\% low-meltingtemperature agarose as indicated in the data. Total well volume was $200 \mu \mathrm{L}$. These cells were incubated for 1.5 hours at $37^{\circ} \mathrm{C}$ at $120^{\circ}$ tilt from horizontal with $200 \mathrm{rpm}$ shaking until they reached the beginning of exponential growth, at which point they were distributed into a 96-well plate, covered with a lid, and put into the plate reader with $0.625 \mu \mathrm{g} / \mathrm{mL}$ tetracycline at $37^{\circ} \mathrm{C}$. OD600 and GFP fluorescent intensity of each well was monitored in the plate reader with measurements taken every 10 minutes, with 10 seconds of orbital shaking (amplitude 3) every minute. Cells were grown in the plate for 1 hour before being diluted 1:100 into PBS. Some of the cells were analyzed using a 487 Novocyte Flow Cytometer (ACEA Biosciences), and some were used for cell sorting for 488 the second phase of the experiment.

489 To measure the switching of cells from an "off" state to an "on" state, cells were sorted 490 in an Astrios Cell Sorter (Beckman Coulter), defining the "off" population with a 491 combination of gates on FITC height and width, thresholding reads on side scatter.

492 Once sorted, 2500 of the "off" cells were put in LB media with $30 \mu \mathrm{g} / \mathrm{mL}$ kanamycin, $493 \quad 0.625 \mu \mathrm{g} / \mathrm{mL}$ tetracycline, and 0.5\% low-melting-temperature agarose for a total well 494 volume of $200 \mu \mathrm{L}$. The plate was covered with a plastic lid and grown for $\sim 12$ hours at 
$37^{\circ} \mathrm{C}$. OD600 and GFP fluorescent intensity of each well was monitored in the plate reader with measurements taken every 10 minutes, with 10 seconds of orbital shaking (amplitude 3) every minute. Once the cells had reached the same cell density that they had achieved in the first hour of growth, $p Z\left(P_{\text {const-TetX-GFP)-containing cells were }}\right.$ diluted 1:100 in PBS and analyzed using a Novocyte Flow Cytometer (ACEA Biosciences).

Data from each sample was analyzed using FCS Express version 4.0 (De Novo Software, Los Angeles, CA). Results were gated by only including counts that had a linear correlation between area and height measurement on the FITC fluorescent channel. The "on" population and the "off" population were separately determined to separate the two modes seen in the data, and these boundaries were uniformly applied to each replicate. For the "off" to "on" measurement, between five thousand and eighteen thousand counts were collected after gating. For the "on" to "off" measurement, between seventeen thousand and thirty-seven thousand counts were collected after gating.

\section{Simulation of stochastic resonance using a stochastic differential equation}

512 Simulation was performed in MATLAB. Equations were entered using the structures

513 represented in Eq.1 or Eq. 2. The stochastic differential equation was implemented

514 using the Euler-Maruyama method. 1000 simulations were done for each condition and

515 averaged together. Average cell growth of each curve with respect to increasing noise

516 was normalized to average cell growth at the lowest noise intensity.

Data and software availability

519 The datasets and simulations generated during and/or analyzed during the current 520 study are available from the corresponding author upon request.

\section{Acknowledgements}

523 The work is supported by Human Frontier Science Program RGY0080-2015 (C.T.) and 524 National Science Foundation CHE1808237 (C.T.). Research reported in this publication 
was supported by the National Institutes of Health under award number S10OD018223.

\section{Author contributions}

528 DL and CT designed the study and wrote the manuscript. TG assisted with the 529 characterization of the constitutive TetX construct, performed the Perceval experiments 530 and the qPCR experiments. YX assisted with the characterization the gel effect on cell 531 growth and the assembly of constructs used in this study.

\section{Competing financial interests}

534 The authors declare no competing financial interests.

\section{Supplementary Figure Legends}

537 Supplementary Figure 1. Frequency spectra of cells for A490/A405 ratio

538 Graphs representing Fourier transformations of the oscillating ratio of absorbance at $539490 \mathrm{~nm}$ and absorbance at $405 \mathrm{~nm}$. The ratio of A490/A405 has been shown to represent 540 the ratio of ATP to ADP in cells expressing Perceval. These results show that the

541 frequency spectra of cells with Perceval and cells without Perceval can be distinguished 542 at $0.0 \%$ and $0.25 \%$ gel, but not $0.5 \%$ or $0.75 \%$ gel. Furthermore, the frequency spectra 543 of cells expressing Perceval and shaken at $10 \mathrm{~s} / \mathrm{min}$ can be distinguished from cells 544 expressing Perceval and shaken at 0s/min. All graphs show 3 biological replicates, 545 except for $\mathrm{DH} 5 \alpha$ cells in $0.0 \%$ gel with $10 \mathrm{~s} /$ min shaking, which shows 2 biological 546 replicates.

548 Supplementary Figure 2. Bacteria expressing a luminescent reporter under periodic 549 and heterogeneous conditions.

550 Metabolic activity of cells in media with .5\% low-melting-temperature agarose as 551 measured by cells expressing luciferase, which uses an ATP- and oxygen-dependent 
552 reaction to catalyze the production of light. Periodic shaking induces an approximately

553 two-fold periodic change in luminescence (Methods- section D). Along with fluctuations

554 in Perceval A490/A405 levels, these results suggest that periodic shaking of cells

555 imparts periodic perturbation of metabolism in those cells. Points represent mean

556 values, and error bars represent standard error of the mean. Mean and SEM are

557 calculated using six biological replicates.

Supplementary Figure 3. Changes in OD600 over time for DH5 $\alpha$ and DH5 $\alpha$ pro cells 560 grown in hydrogel and tetracycline.

561 (A) Changes in OD600 over time for DH5 $\alpha$ grown without shaking.

562 (B) Changes in OD600 over time for $\mathrm{DH} 5 \alpha$ grown with 10 seconds shaking per minute.

563 (C) Changes in OD600 over time for DH5 $\alpha$ pro grown without shaking.

564 (D) Changes in OD600 over time for DH5 $\alpha$ pro grown with 10 seconds shaking per 565 minute.

566 (A-D) Cell grown in LB with $0,0.25,0.5$, or $0.75 \%$ gel along with $0 \mu \mathrm{g} / \mathrm{mL}, 0.3125$ $567 \mu \mathrm{g} / \mathrm{mL}$, or $0.625 \mu \mathrm{g} / \mathrm{mL}$ tetracycline. Points represent mean values, and error bars 568 represent standard error of the mean. Mean and SEM are calculated using six biological 569 replicates.

571 Supplementary Figure 4. Hydrogel encapsulation causes heterogeneous protein 572 expression.

573 (A) Histograms of GFP intensity of DH5 $\alpha$ cells constitutively expressing TetX-GFP in LB 574 with $0 \%$ gel. All biological replicates are shown.

575 (B) Histograms of GFP intensity of $\mathrm{DH} 5 \alpha$ cells constitutively expressing TetX-GFP in LB 576 with $0.5 \%$ gel. All biological replicates are shown.

577 (A\&B) Histograms represent 5000-8000 events after filtering. 
580 Supplementary Figure 5: Periodic perturbation does not increase gene module expression.

582 Fluorescent intensity of TetX-GFP normalized by OD600 of cells after 1 hour of growth. 583 Two-tailed, equal variance t-test returns $p$-value $=0.345$ N.S. stands for not significant. 584 Bar height represents mean values, and error bars represent standard error of the 585 mean. Mean and SEM are calculated using eight biological replicates.

Supplementary Figure 6. Significance testing of relative growth curves.

(A) Confidence intervals of relative growth for bacteria constitutively expressing stable

TetX grown with $0 \mathrm{~s} / \mathrm{min}$ shaking (left panel) $0 \mu \mathrm{g} / \mathrm{mL}$ tetracycline (middle panel) 0.3125 $\mu \mathrm{g} / \mathrm{mL}$ tetracycline (right panel) $0.625 \mu \mathrm{g} / \mathrm{mL}$ tetracycline

(B) Confidence intervals of relative growth for bacteria constitutively expressing stable

594 (C) Confidence intervals of relative growth for bacteria constitutively expressing stable

(D) Confidence intervals of relative growth for bacteria inducibly expressing stable TetX 598 grown with $0 \mathrm{~s} / \mathrm{min}$ shaking (left panel) $0 \mu \mathrm{g} / \mathrm{mL}$ tetracycline (middle panel) 0.3125 $599 \mu \mathrm{g} / \mathrm{mL}$ tetracycline (right panel) $0.625 \mu \mathrm{g} / \mathrm{mL}$ tetracycline

600 (E) Confidence intervals of relative growth for bacteria inducibly expressing stable TetX 601 grown with $10 \mathrm{~s} / \mathrm{min}$ shaking (left panel) $0 \mu \mathrm{g} / \mathrm{mL}$ tetracycline (middle panel) 0.3125 $602 \mu \mathrm{g} / \mathrm{mL}$ tetracycline (right panel) $0.625 \mu \mathrm{g} / \mathrm{mL}$ tetracycline

603 (F) Confidence intervals of relative growth for bacteria inducibly expressing stable TetX 604 grown with $50 \mathrm{~s} / \mathrm{min}$ shaking (left panel) $0 \mu \mathrm{g} / \mathrm{mL}$ tetracycline (middle panel) 0.3125 
$605 \mu \mathrm{g} / \mathrm{mL}$ tetracycline (right panel) $0.625 \mu \mathrm{g} / \mathrm{mL}$ tetracycline

606 (A-F) Significance intervals calculated by Bonferroni multiple comparisons test within 607 the growth curve (Methods- Section G).

608

609 Supplementary Figure 7. Changes in relative growth over time for DH5 $\alpha$ cells expressing TetX grown in hydrogel and tetracycline.

611 (A) Changes in relative growth over time for $\mathrm{DH} 5 \alpha$ cells expressing TetX constitutively, 612 grown without shaking.

613 (B) Changes in relative growth over time for DH5 $\alpha$ cells expressing TetX constitutively, 614 grown with $10 \mathrm{~s} / \mathrm{min}$ shaking. Large reading at an early time point of $0.75 \%$ gel and 0 $615 \mu \mathrm{g} / \mathrm{mL}$ tetracycline correspond to a bubble interfering with reading.

616 (C) Changes in relative growth over time for $\mathrm{DH} 5 \alpha$ cells expressing TetX constitutively, 617 grown with 50 s/min shaking.

618 (D) Changes in relative growth over time for DH5 $\alpha$ cells expressing TetX-ssra 619 constitutively, grown without shaking. Degradation tag (ssra) makes TetX unstable.

620 (E) Changes in relative growth over time for DH5 $\alpha$ cells expressing TetX-ssra 621 constitutively, grown with 10s/min shaking. Degradation tag (ssra) makes TetX 622 unstable.

623 (A-E) Cell grown in LB with $0,0.25,0.5$, or $0.75 \%$ gel along with $0 \mu \mathrm{g} / \mathrm{mL}, 0.3125$ $624 \mu \mathrm{g} / \mathrm{mL}$, or $0.625 \mu \mathrm{g} / \mathrm{mL}$ tetracycline. Points represent mean values, and error bars 625 represent standard error of the mean. Mean and SEM are calculated using six biological 626 replicates except for $(C)$, which is calculated using four biological replicates.

628 Supplementary Figure 8. Inducible circuit characterization.

629 Characterization of $\mathrm{P}_{\text {Tet }}$ producing GFP in DH5 $\alpha$ pro cells in response to anhydrous 630 tetracycline. Cell line only control is background fluorescence of DH5 $\alpha$ pro cells. 
631 Points represent individual replicates, line highlights trend. There are four replicates per 632 condition.

634 Supplementary Figure 9. Changes in OD600 over time for DH5 $\alpha$ pro cells expressing 635 TetX grown in hydrogel and tetracycline.

636 (A) Changes in relative growth over time for DH5 $\alpha$ pro cells expressing TetX upon 637 tetracycline induction, grown without shaking.

638 (B) Changes in relative growth over time for DH5 $\alpha$ pro cells expressing TetX upon 639 tetracycline induction, grown with 10s/min shaking.

640 (C) Changes in relative growth over time for $\mathrm{DH} 5 \alpha$ pro cells expressing TetX 641 constitutively, grown with 50 s/min shaking.

642 (D) Changes in relative growth over time for DH5 $\alpha$ pro cells expressing TetX-ssra upon 643 tetracycline induction, grown without shaking. Degradation tag (ssra) makes TetX 644 unstable.

645 (E) Changes in relative growth over time for DH5 $\alpha$ pro cells expressing TetX-ssra upon 646 tetracycline induction, grown with 10s/min shaking. Degradation tag (ssra) makes TetX 647 unstable.

648 (A-E) Cell grown in LB with $0,0.25,0.5$, or $0.75 \%$ gel along with $0 \mu \mathrm{g} / \mathrm{mL}, 0.3125$ $649 \mu \mathrm{g} / \mathrm{mL}$, or $0.625 \mu \mathrm{g} / \mathrm{mL}$ tetracycline. Points represent mean values, and error bars 650 represent standard error of the mean. Mean and SEM are calculated using six biological 651 replicates. 


\section{REFERENCES}

655 Alnahhas, R.N., Winkle, J.J., Hirning, A.J., Karamched, B., Ott, W., Josic, K., and Bennett, M.R.

656 (2019). Spatiotemporal Dynamics of Synthetic Microbial Consortia in Microfluidic Devices. ACS 657 synthetic biology 8, 2051-2058.

658 Alvarez, G.S., Foglia, M.L., Copello, G.J., Desimone, M.F., and Diaz, L.E. (2009). Effect of 659 various parameters on viability and growth of bacteria immobilized in sol-gel-derived silica 660 matrices. Appl Microbiol Biot 82, 639-646.

661 Balasubramanian, S., Aubin-Tam, M.E., and Meyer, A.S. (2019). 3D Printing for the Fabrication of Biofilm-Based Functional Living Materials. ACS synthetic biology 8, 1564-1567.

663 Berg, J., Hung, Y.P., and Yellen, G. (2009). A genetically encoded fluorescent reporter of 664 ATP:ADP ratio. Nat Methods 6, 161-166.

665 Biselli, E., Schink, S.J., and Gerland, U. (2021). Slower growth of Escherichia coli leads to 666 longer survival in carbon starvation due to a decrease in the maintenance rate (vol 16, e9478, 667 2020). Mol Syst Biol 17.

668 Bittihn, P., Didovyk, A., Tsimring, L.S., and Hasty, J. (2020). Genetically engineered control of 669 phenotypic structure in microbial colonies. Nature microbiology 5, 697-705.

670 Cao, Y., Feng, Y., Ryser, M.D., Zhu, K., Herschlag, G., Cao, C., Marusak, K., Zauscher, S., and 671 You, L. (2017). Programmable assembly of pressure sensors using pattern-forming bacteria.

672 Nature biotechnology 35, 1087-1093.

673 Cao, Y., Ryser, M.D., Payne, S., Li, B., Rao, C.V., and You, L. (2016). Collective Space-

674 Sensing Coordinates Pattern Scaling in Engineered Bacteria. Cell 165, 620-630.

675 Chyzy, A., and Plonska-Brzezinska, M.E. (2020). Hydrogel Properties and Their Impact on 676 Regenerative Medicine and Tissue Engineering. Molecules 25.

677 Dal Co, A., van Vliet, S., Kiviet, D.J., Schlegel, S., and Ackermann, M. (2020). Short-range 678 interactions govern the dynamics and functions of microbial communities. Nature ecology \& 679 evolution 4, 366-375.

680 Deris, J.B., Kim, M., Zhang, Z., Okano, H., Hermsen, R., Groisman, A., and Hwa, T. (2013). The 681 innate growth bistability and fitness landscapes of antibiotic-resistant bacteria. Science 342, 6821237435.

683 Dufrene, Y.F., and Persat, A. (2020). Mechanomicrobiology: how bacteria sense and respond to 684 forces. Nature reviews Microbiology 18, 227-240.

685 Gammaitoni, L., Hanggi, P., Jung, P., and Marchesoni, F. (1998). Stochastic resonance. Rev 686 Mod Phys 70, 223-287.

687 Ghosh, S., LaPara, T.M., and Sadowsky, M.J. (2015). Transformation of tetracycline by TetX 
and its subsequent degradation in a heterologous host. FEMS microbiology ecology 91 . Nature materials 20,691-700.

692 Gonzalez, L.M., Mukhitov, N., and Voigt, C.A. (2020). Resilient living materials built by printing 693 bacterial spores. Nature chemical biology 16, 126-133.

694 Gottesman, S., Roche, E., Zhou, Y., and Sauer, R.T. (1998). The ClpXP and ClpAP proteases 695 degrade proteins with carboxy-terminal peptide tails added by the SsrA-tagging system. Genes 696 Dev 12, 1338-1347.

697 Gupta, S., Ross, T.D., Gomez, M.M., Grant, J.L., Romero, P.A., and Venturelli, O.S. (2020). 698 Investigating the dynamics of microbial consortia in spatially structured environments. Nature 699 communications 11, 2418.

700 Hay, J.J., Rodrigo-Navarro, A., Petaroudi, M., Bryksin, A.V., Garcia, A.J., Barker, T.H., Dalby, M.J., and Salmeron-Sanchez, M. (2018). Bacteria-Based Materials for Stem Cell Engineering. Advanced materials 30, e1804310. between bacteria and hydrogels. Scientific reports 8, 10893. Turing patterns in a synthetic bacterial population. Proceedings of the National Academy of Sciences of the United States of America 115, 6572-6577. structures with synthetic gene circuits. Nucleic acids research 45, 1005-1014.

712 Lopatkin, A.J., Stokes, J.M., Zheng, E.J., Yang, J.H., Takahashi, M.K., You, L., and Collins, J.J.

713 (2019). Bacterial metabolic state more accurately predicts antibiotic lethality than growth rate.

714 Nature microbiology 4, 2109-2117.

715 Manjula-Basavanna, A., Duraj-Thatte, A.M., and Joshi, N.S. (2021). Robust Self-Regeneratable 716 Stiff Living Materials Fabricated from Microbial Cells. Advanced functional materials 31.

717 McDonnell, M.D., and Abbott, D. (2009). What Is Stochastic Resonance? Definitions,

718 Misconceptions, Debates, and Its Relevance to Biology. Plos Comput Biol 5.

719 Nguyen, P.Q., Courchesne, N.D., Duraj-Thatte, A., Praveschotinunt, P., and Joshi, N.S. (2018).

720 Engineered Living Materials: Prospects and Challenges for Using Biological Systems to Direct

721 the Assembly of Smart Materials. Advanced materials 30, e1704847. 
722 Pabst, B., Pitts, B., Lauchnor, E., and Stewart, P.S. (2016). Gel-Entrapped Staphylococcus

723 aureus Bacteria as Models of Biofilm Infection Exhibit Growth in Dense Aggregates, Oxygen

724 Limitation, Antibiotic Tolerance, and Heterogeneous Gene Expression. Antimicrob Agents Ch

$72560,6294-6301$.

726 Paulsson, J., Berg, O.G., and Ehrenberg, M. (2000). Stochastic focusing: fluctuation-enhanced

727 sensitivity of intracellular regulation. Proceedings of the National Academy of Sciences of the

728 United States of America 97, 7148-7153.

729 Sanfilippo, J.E., Lorestani, A., Koch, M.D., Bratton, B.P., Siryaporn, A., Stone, H.A., and Gitai, 730 Z. (2019). Microfluidic-based transcriptomics reveal force-independent bacterial rheosensing.

731 Nature microbiology 4, 1274-1281.

732 Schink, S.J., Biselli, E., Ammar, C., and Gerland, U. (2019). Death Rate of E. coli during

733 Starvation Is Set by Maintenance Cost and Biomass Recycling. Cell systems 9, 64-+.

734 Shao, X.X., Mugler, A., Kim, J., Jeong, H.J., Levin, B.R., and Nemenman, I. (2017). Growth of

735 bacteria in 3-d colonies. Plos Comput Biol 13.

736 Tan, C., Marguet, P., and You, L. (2009). Emergent bistability by a growth-modulating positive

737 feedback circuit. Nature chemical biology 5, 842-848.

738 Taute, K.M., Gude, S., Tans, S.J., and Shimizu, T.S. (2015). High-throughput 3D tracking of

739 bacteria on a standard phase contrast microscope. Nature communications 6, 8776.

740 van Vliet, S., Dal Co, A., Winkler, A.R., Spriewald, S., Stecher, B., and Ackermann, M. (2018).

741 Spatially Correlated Gene Expression in Bacterial Groups: The Role of Lineage History, Spatial

742 Gradients, and Cell-Cell Interactions. Cell systems 6, 496-507 e496.

743 Wessel, A.K., Hmelo, L., Parsek, M.R., and Whiteley, M. (2013). Going local: technologies for

744 exploring bacterial microenvironments. Nature reviews Microbiology 11, 337-348.

745 Zeng, C.H., Zhou, X.F., and Tao, S.F. (2009). Stochastic Resonance in a Bacterium Growth

746 System Subjected to Colored Noises. Commun Theor Phys 52, 615-618.

747 Zhang, R., Goetz, H., Melendez-Alvarez, J., Li, J., Ding, T., Wang, X., and Tian, X.J. (2021).

748 Winner-takes-all resource competition redirects cascading cell fate transitions. Nature

749 communications $12,853$. 
Figure 2

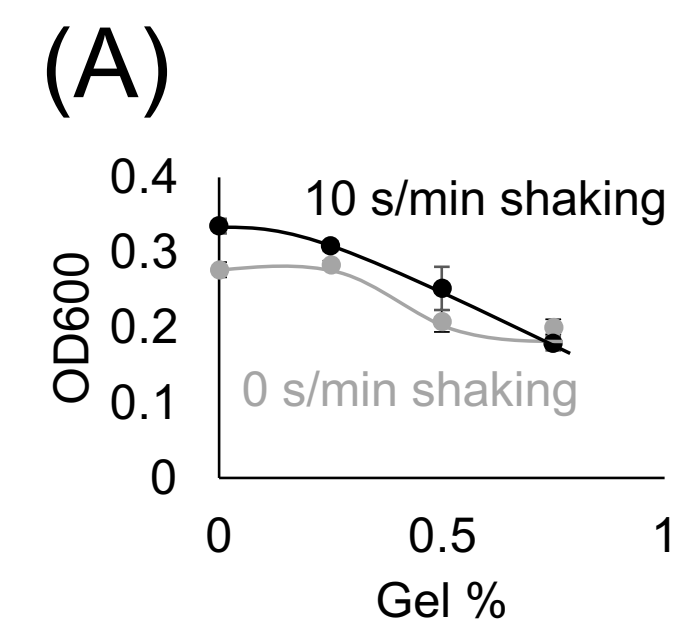

(D)

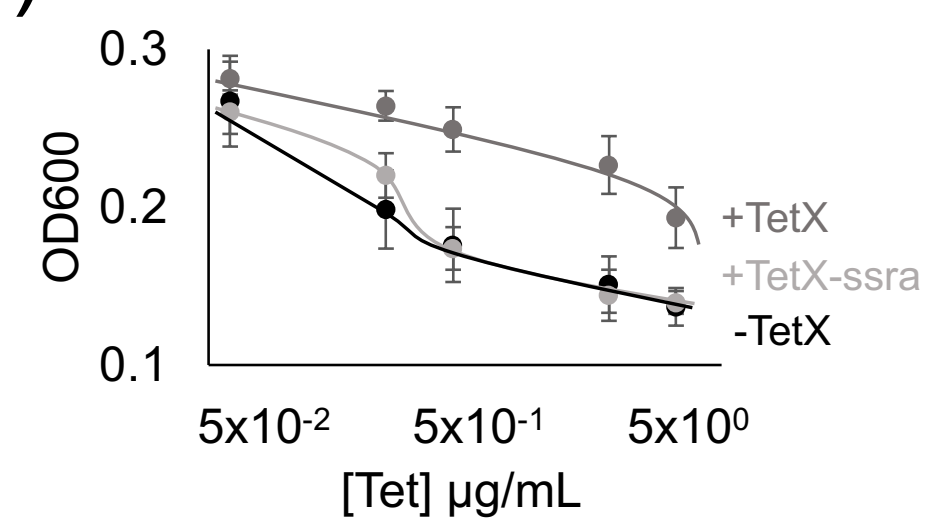

(B)

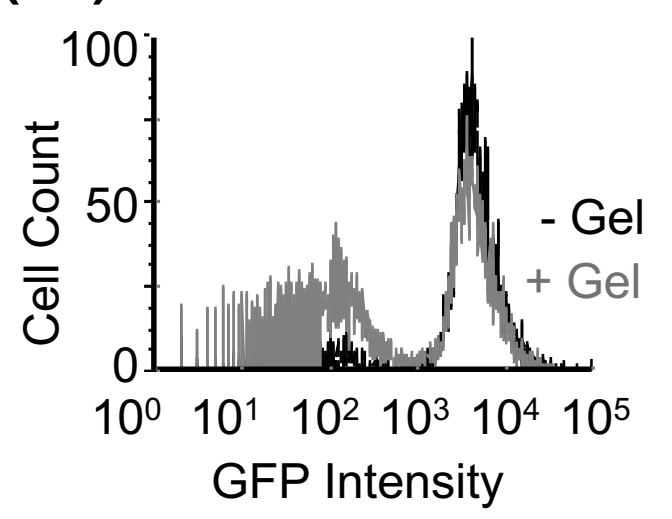

(C)

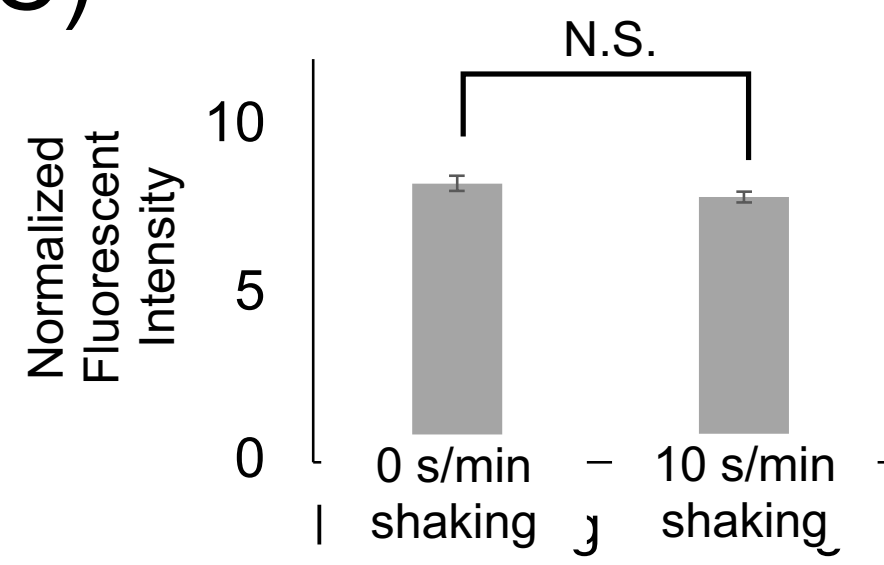

(E)

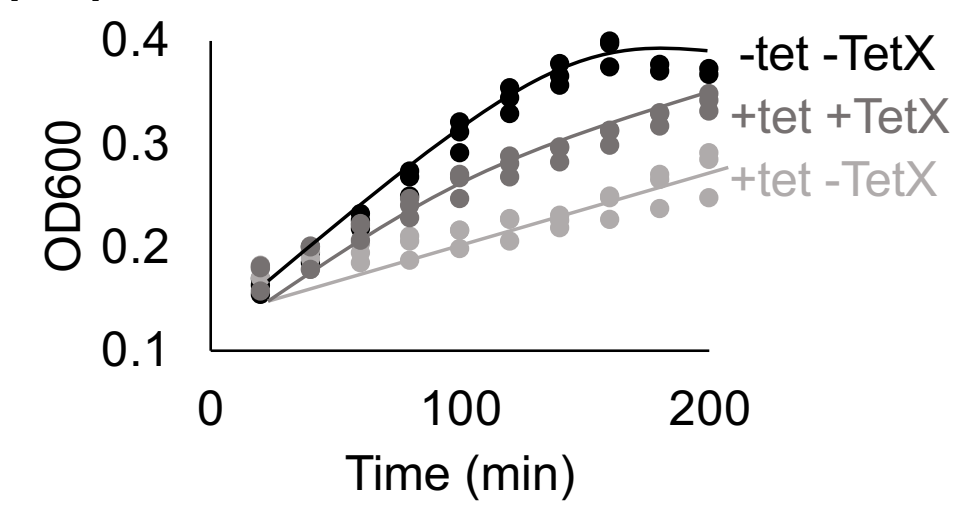




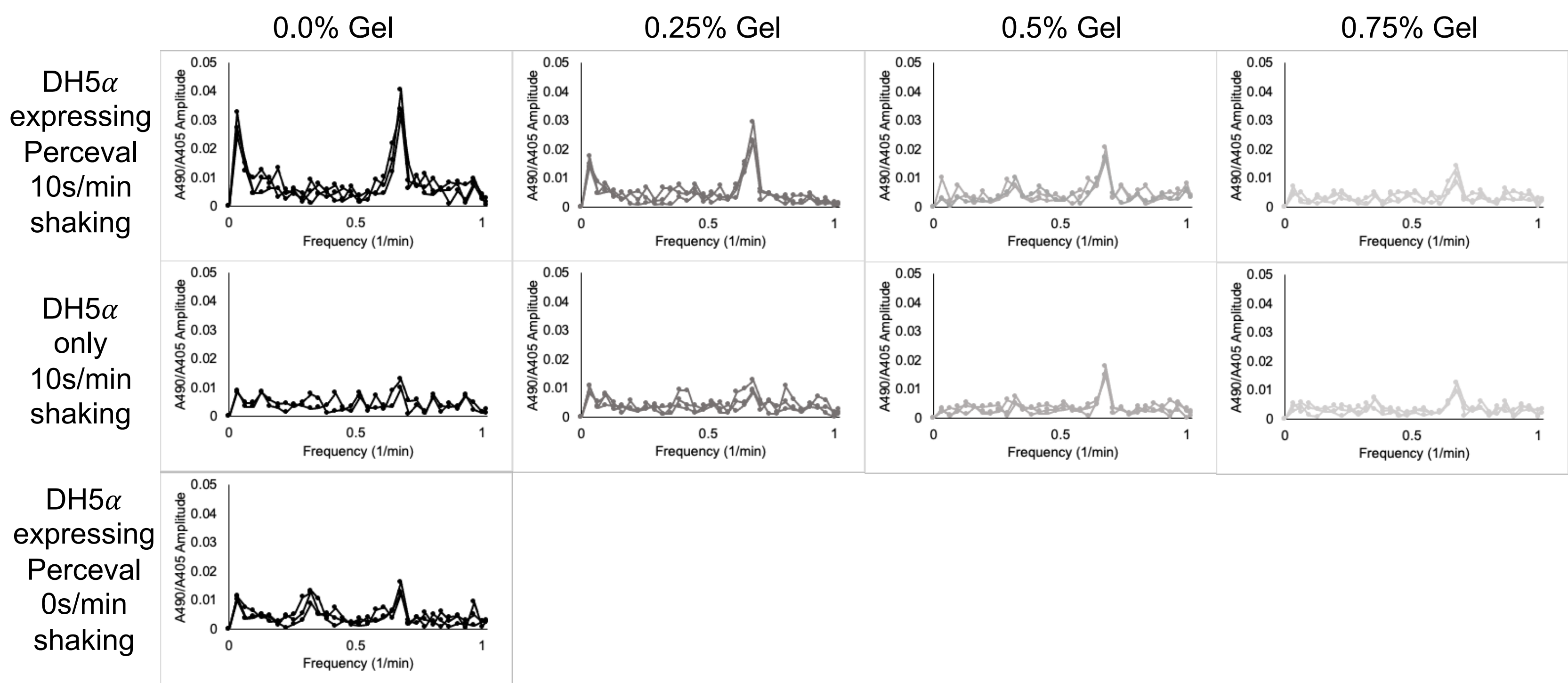


Supplementary Figure 2

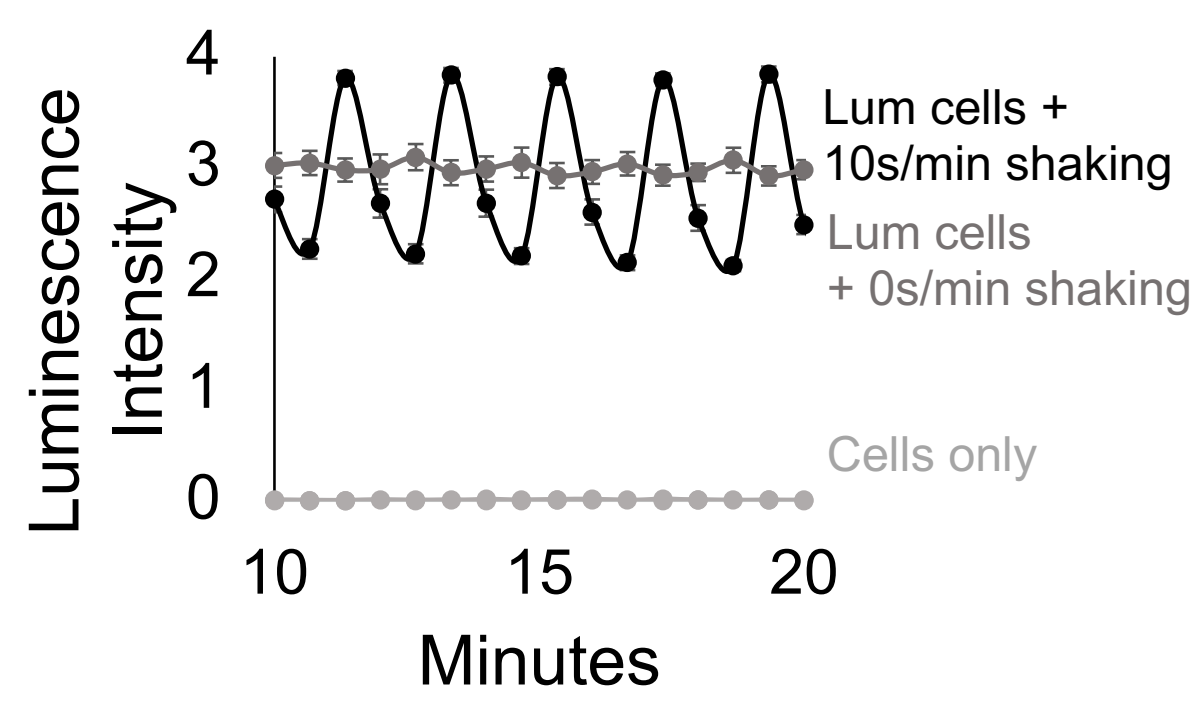




\section{Supplementary Figure 3}

(A)
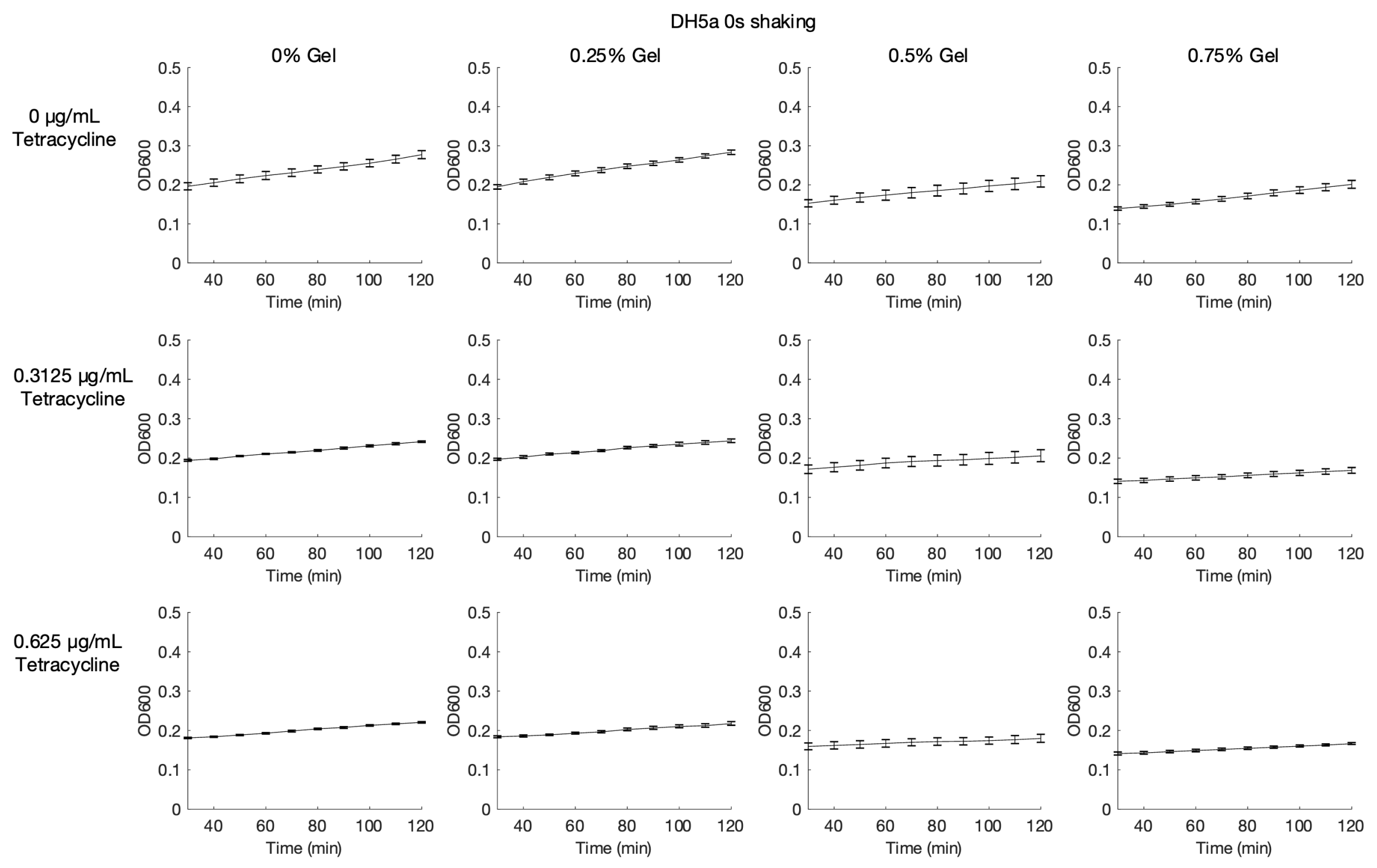


\section{Supplementary Figure 3 - continue}

(B)

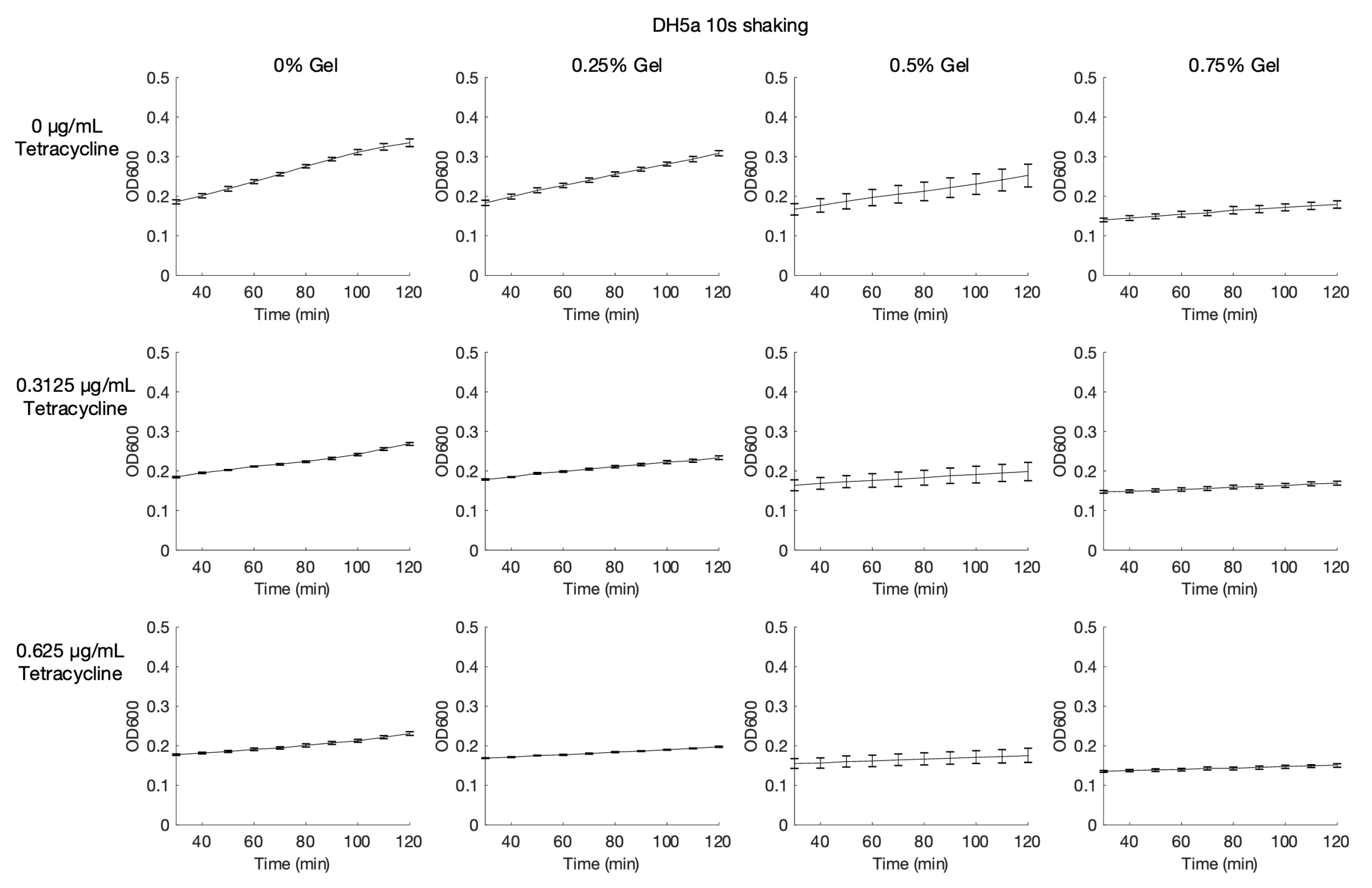




\section{Supplementary Figure 3 - continue}

(C)
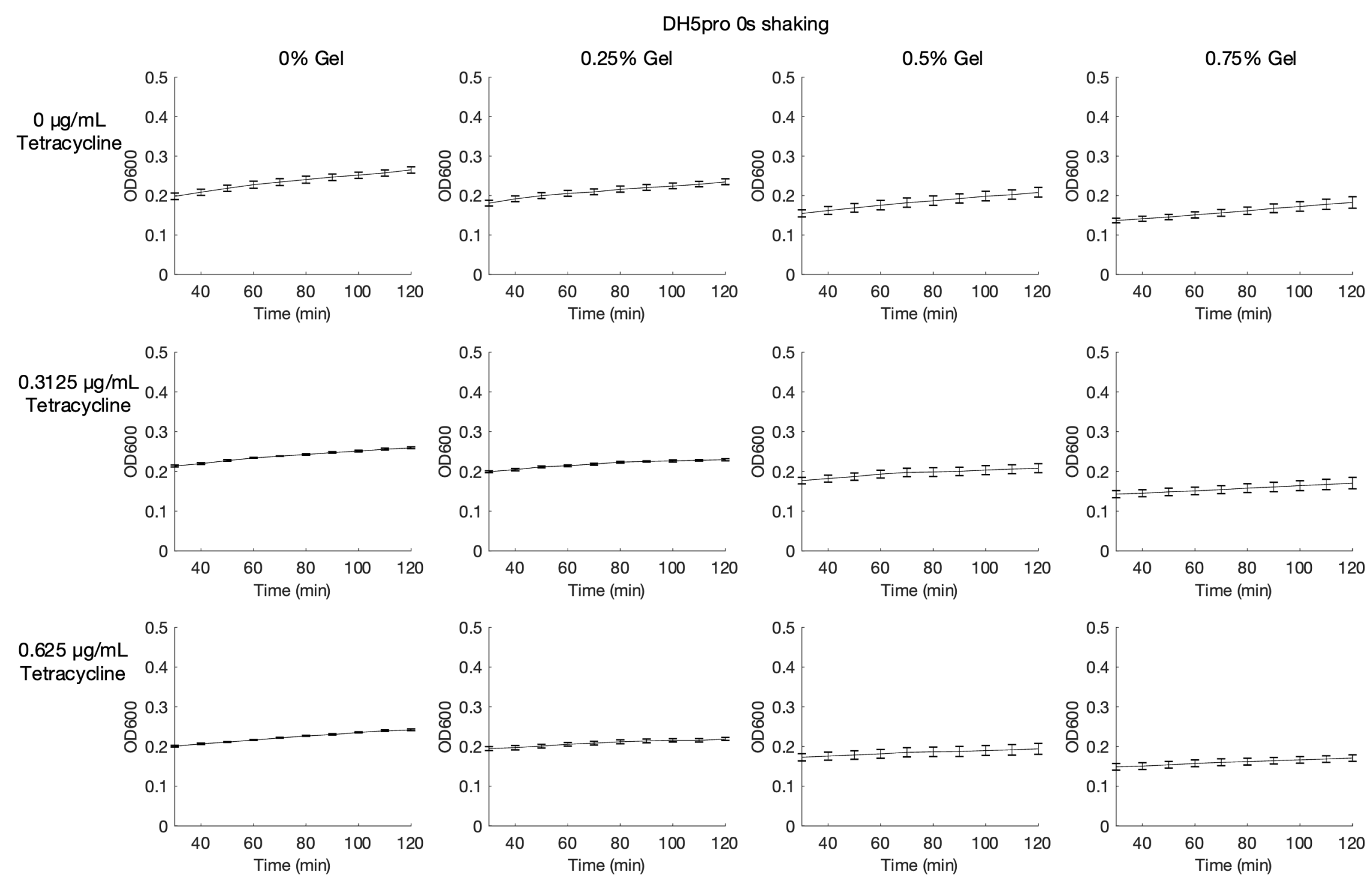


\section{Supplementary Figure 3 - continue}

(D)
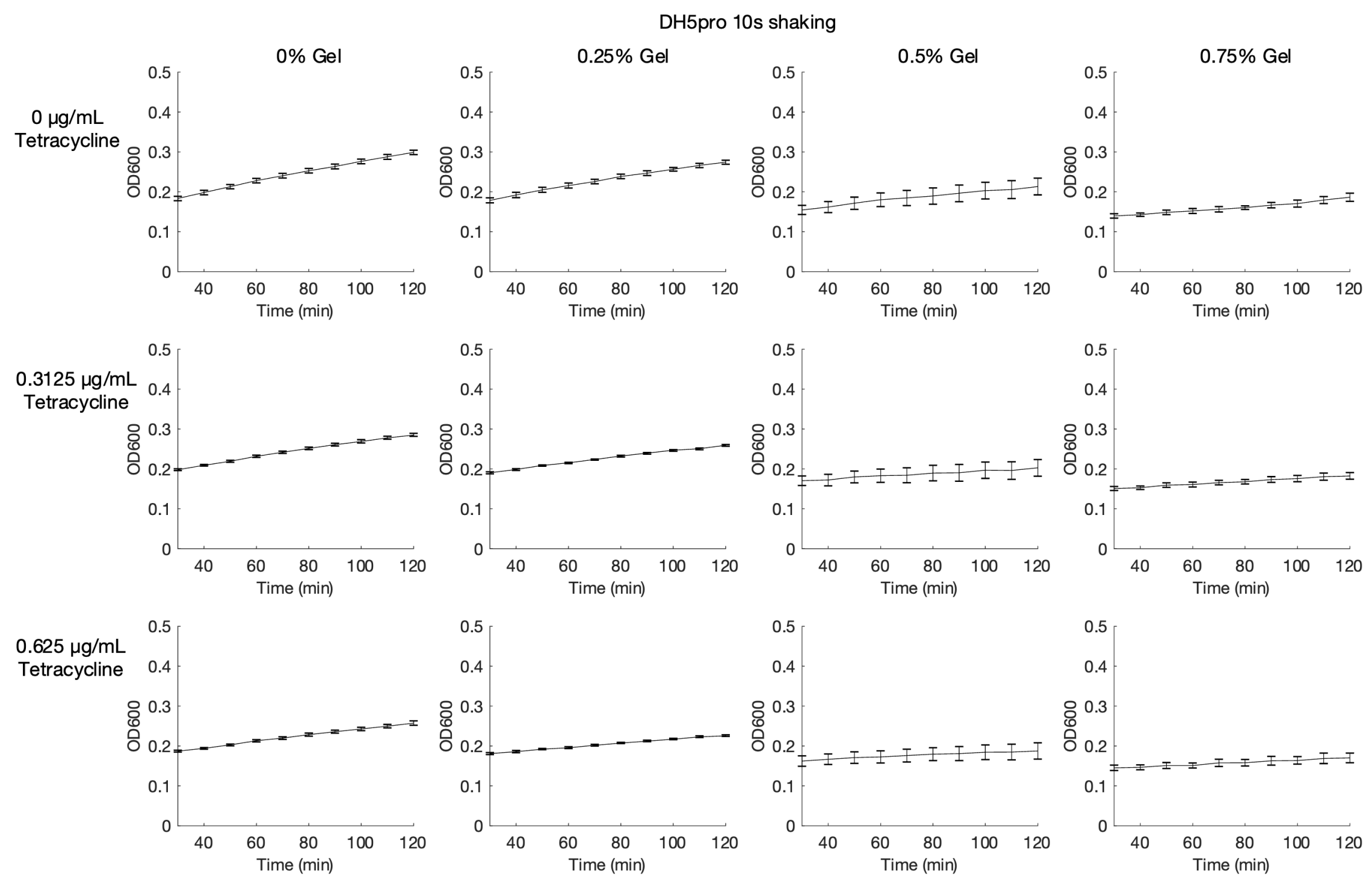
(A) $\mathrm{DH} 5 \alpha$ expressing TetXGFP in $0.0 \%$ Gel in LB

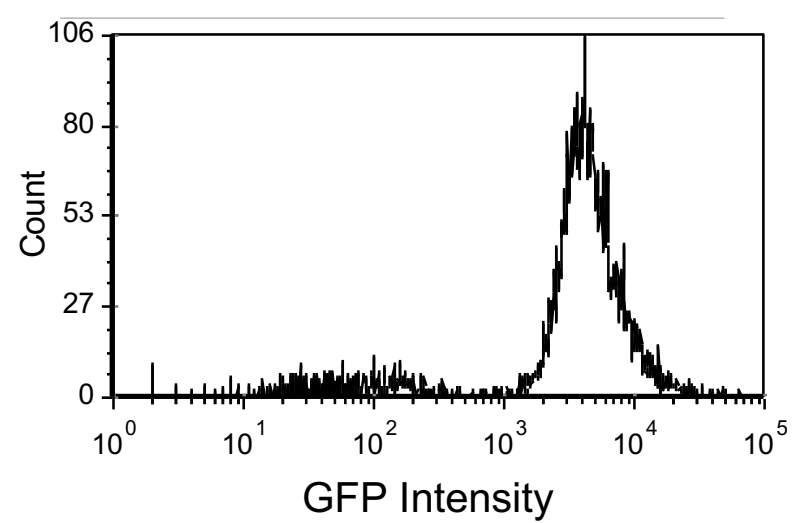

(B) DH5 $\alpha$ expressing TetXGFP in $0.5 \%$ Gel in LB
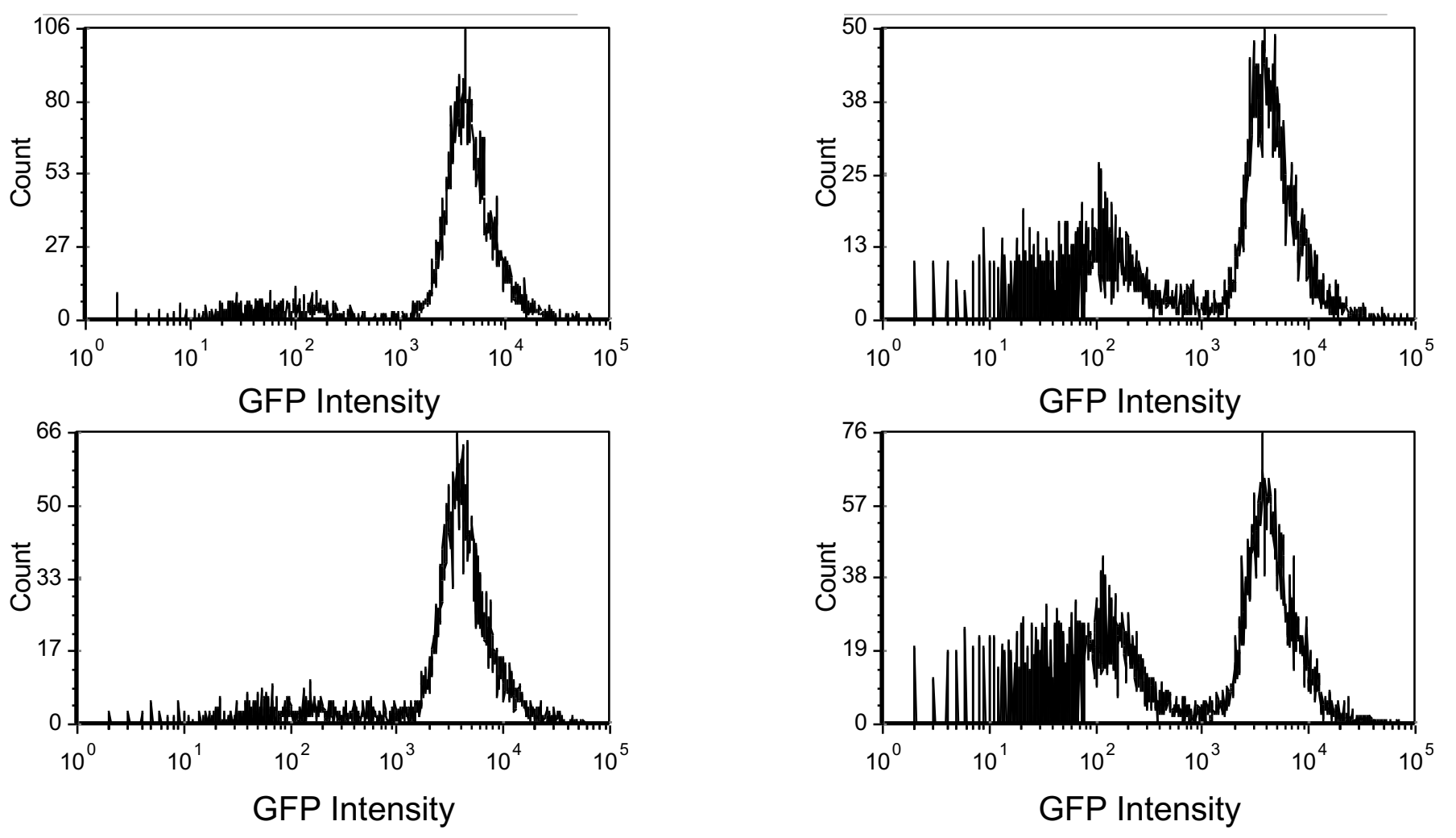


\section{Supplementary Figure 5}

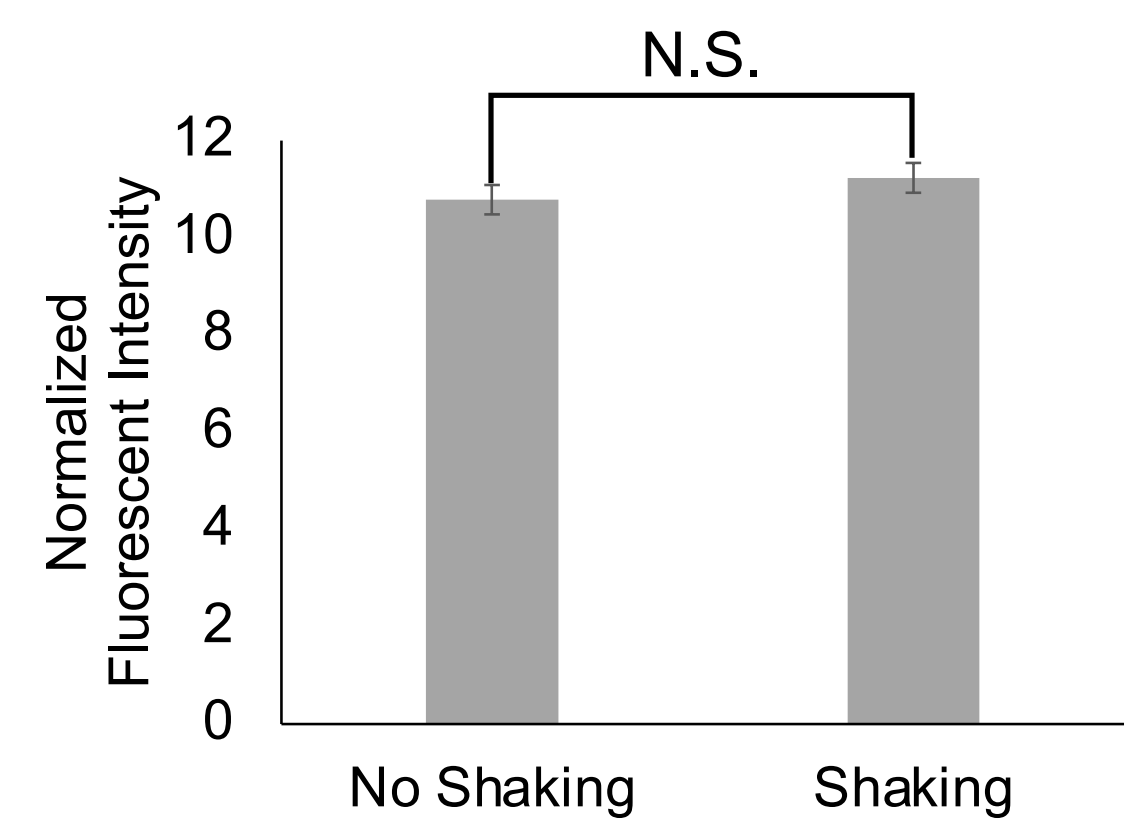




\section{Supplementary Figure 6}

(A)

(B)
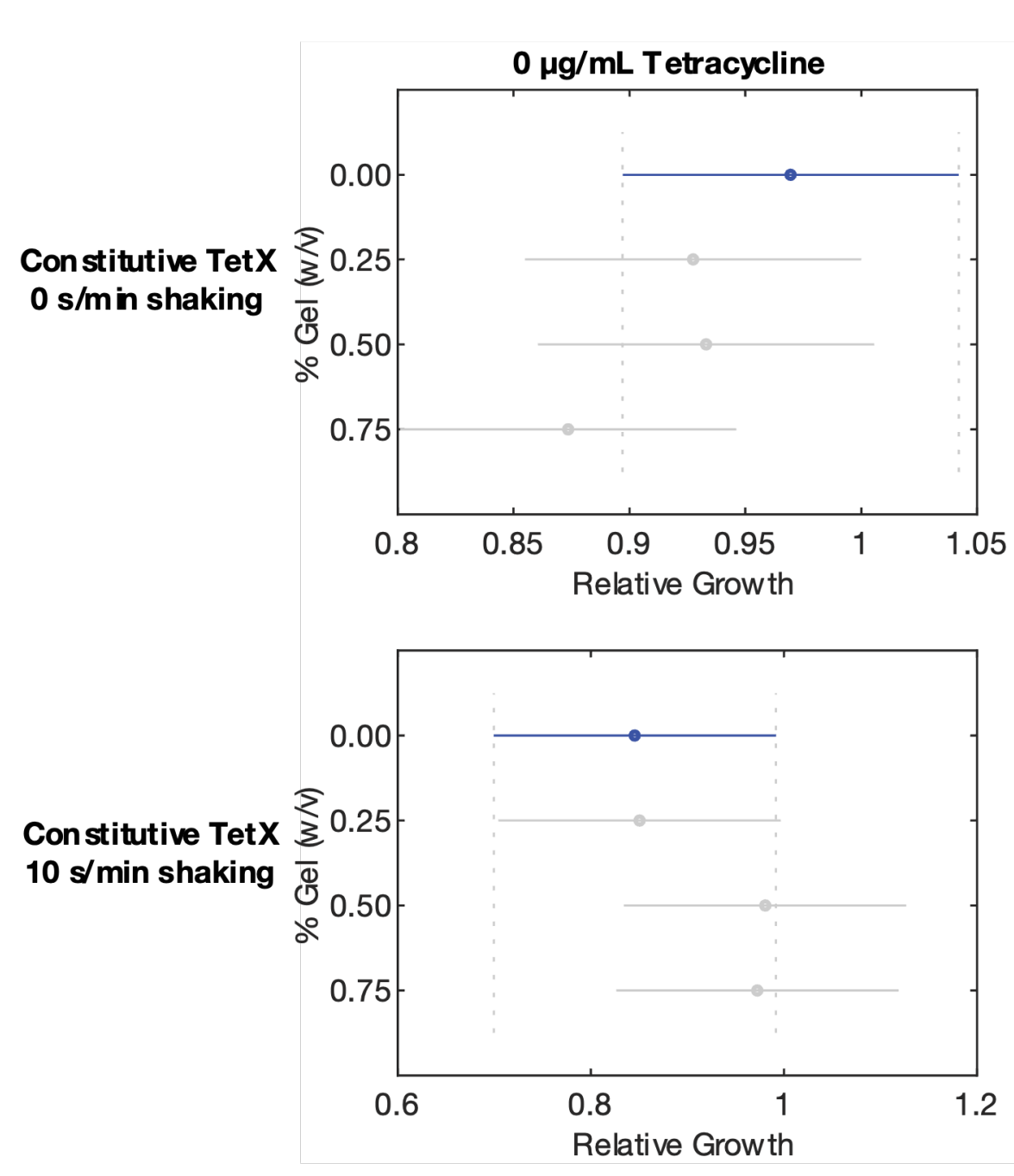
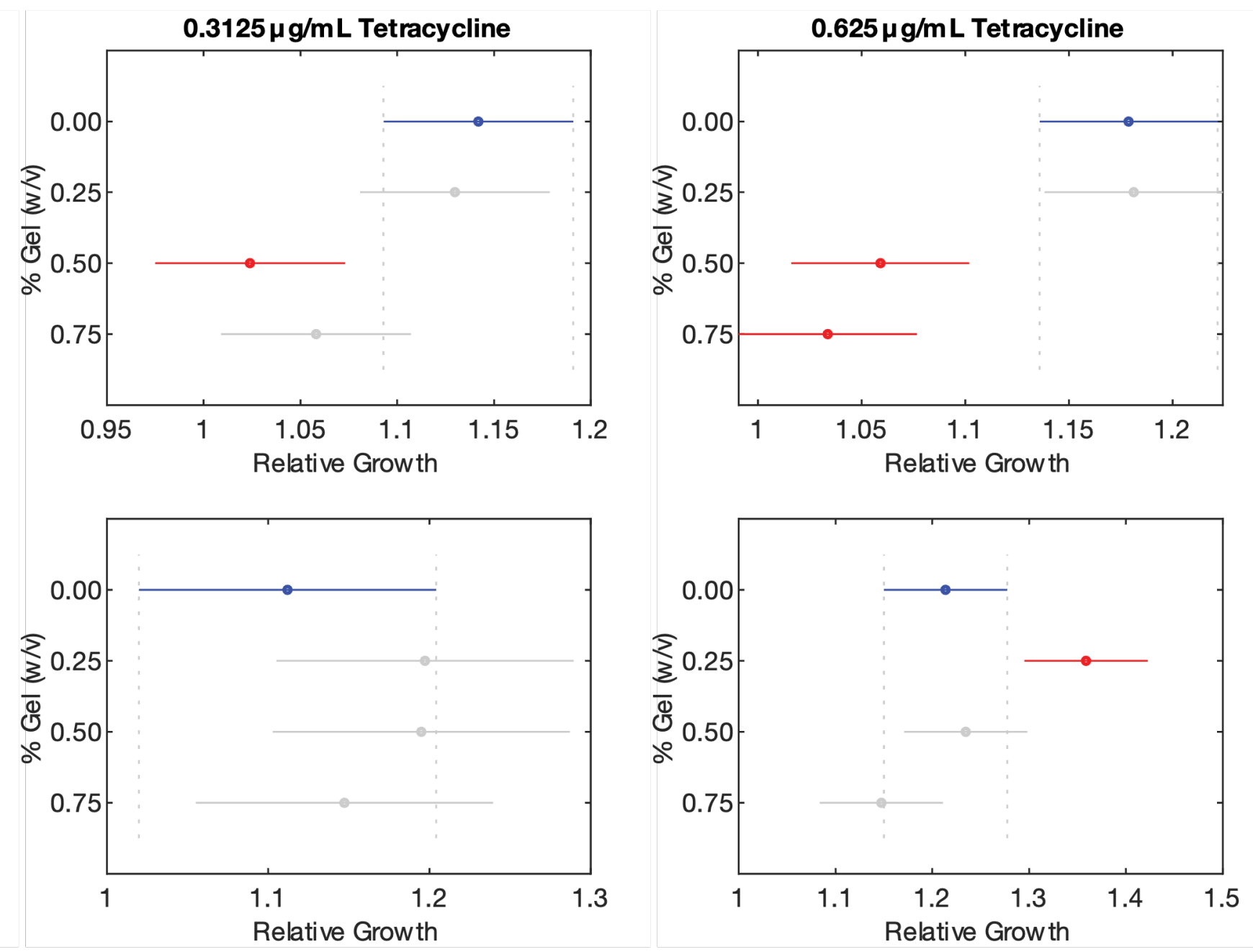


\section{Supplementary Figure 6}

\section{(C)}

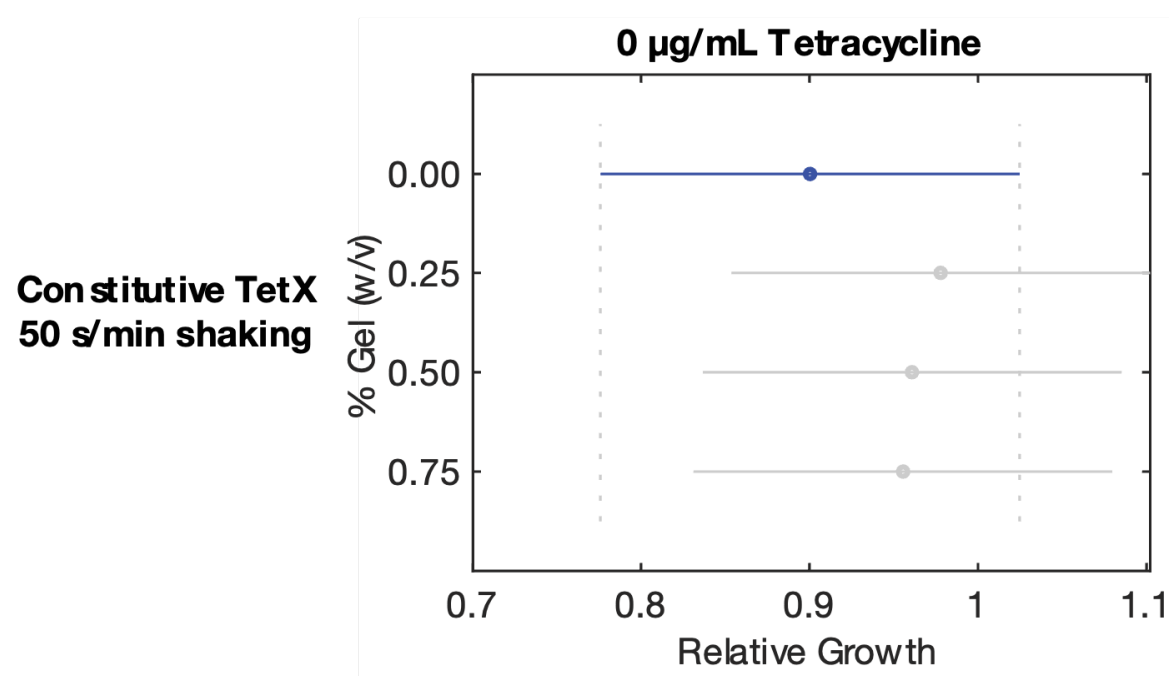

(D)

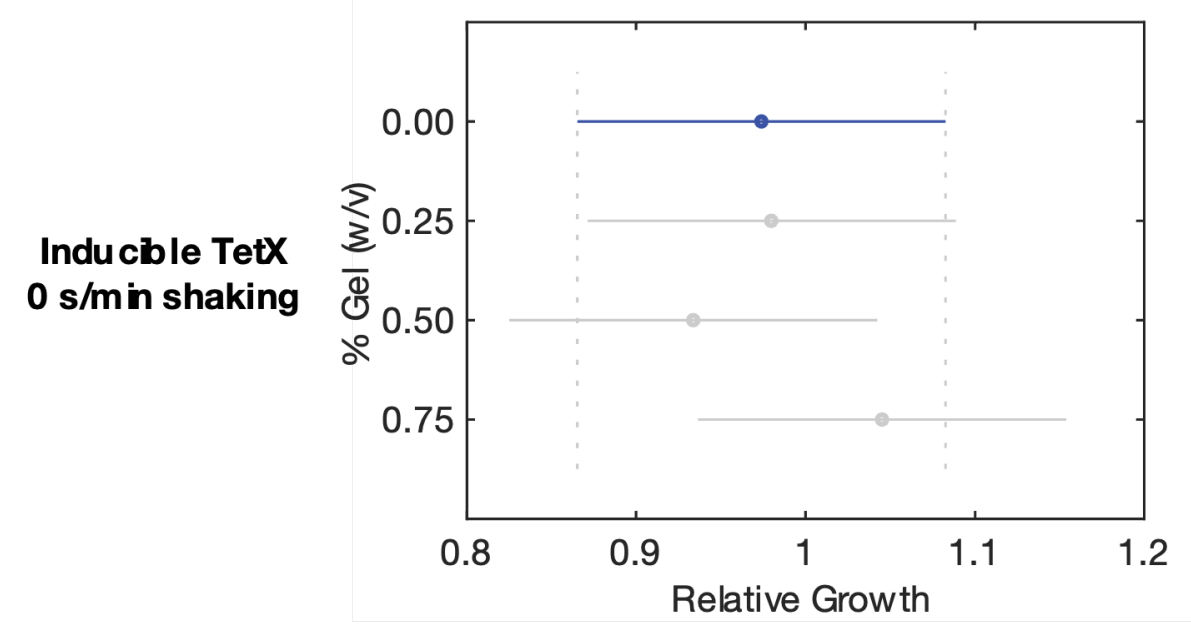

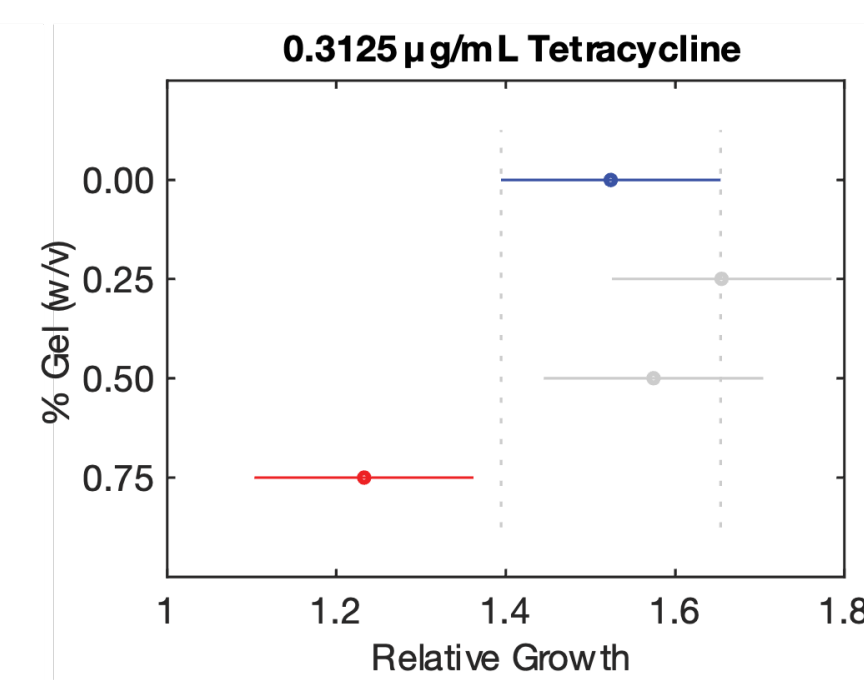
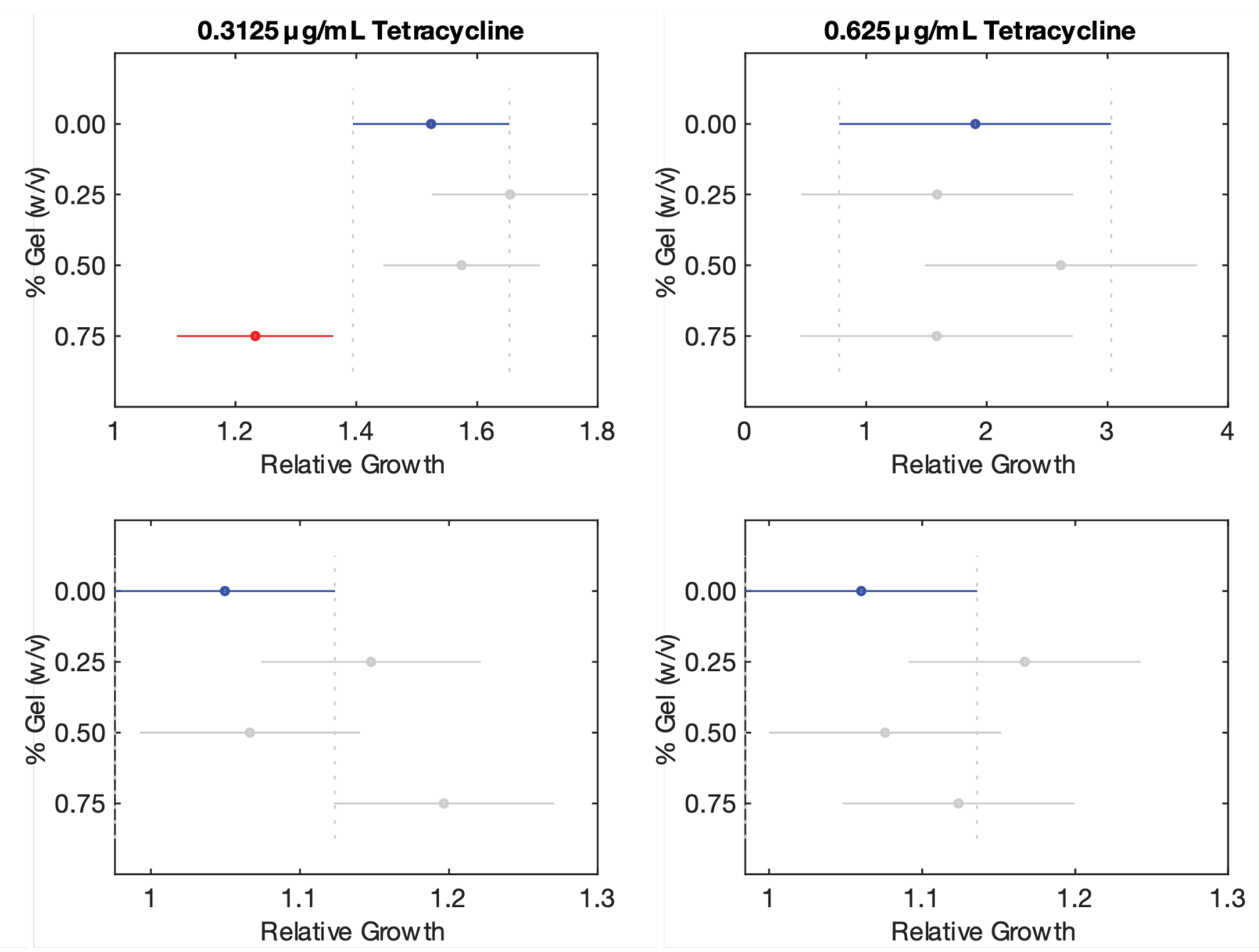


\section{Supplementary Figure 6}

$(\mathrm{E})$

(F)
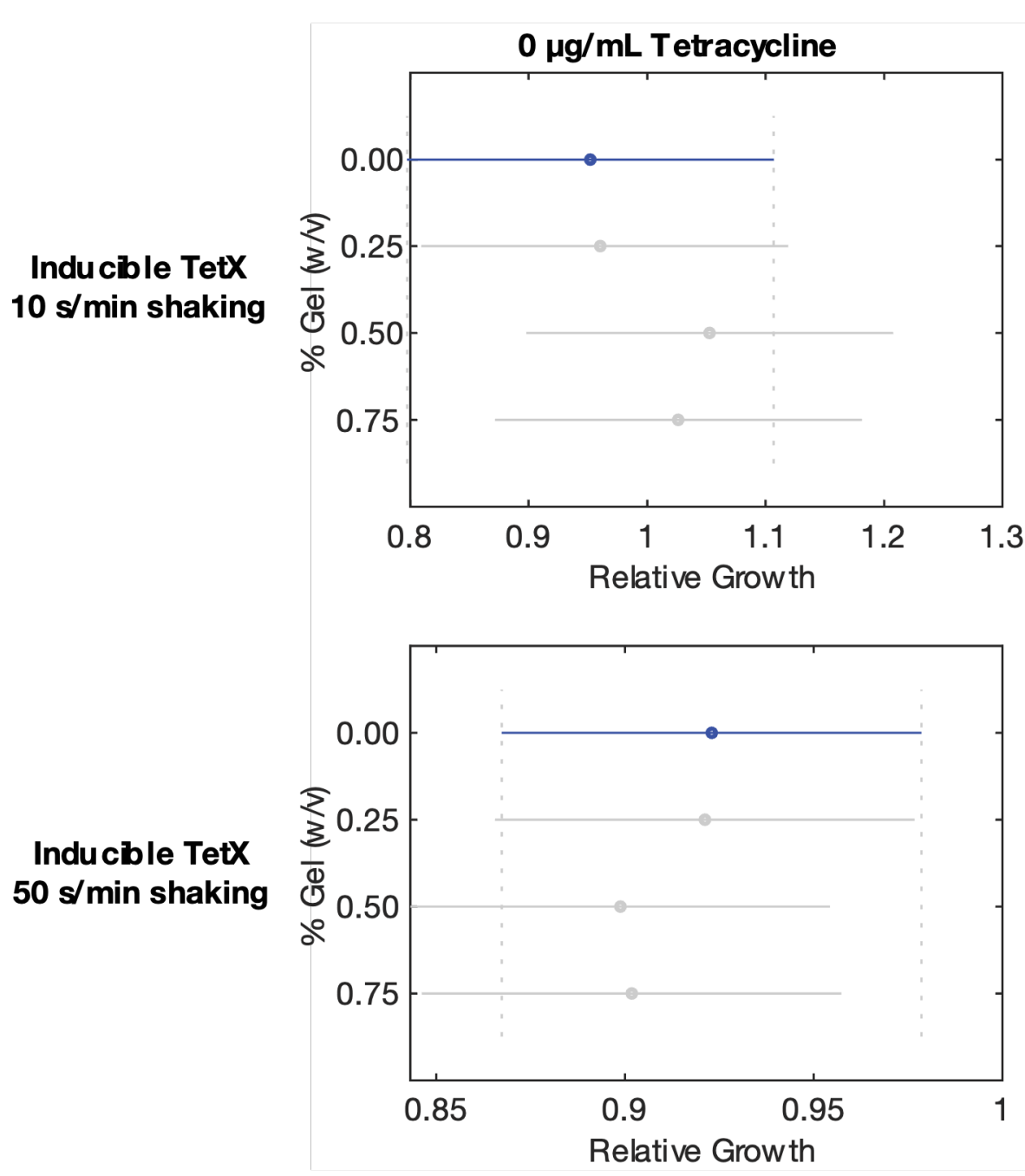

$0.3125 \mu \mathrm{g} / \mathrm{mL}$ Tetracycline
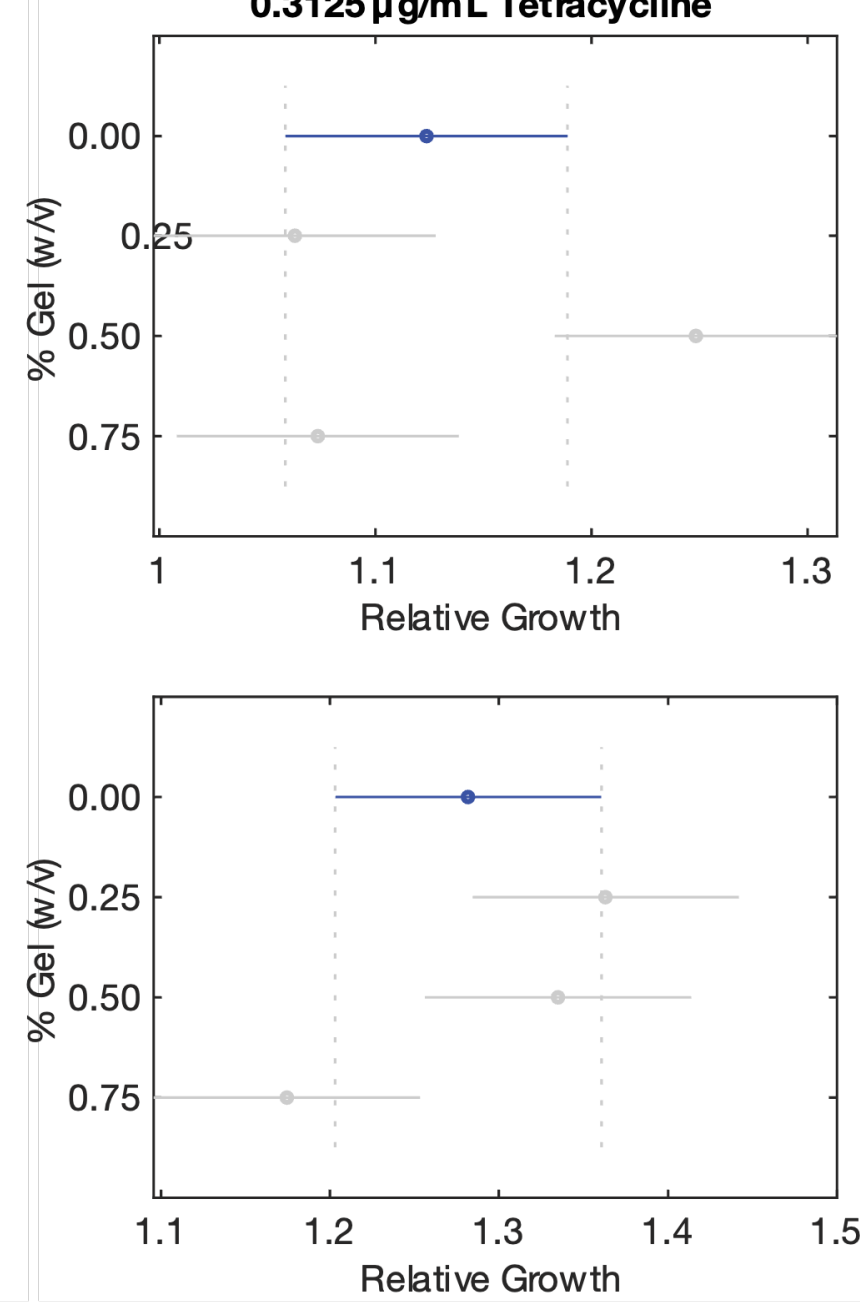

$0.625 \mu \mathrm{g} / \mathrm{mL}$ Tetracycline
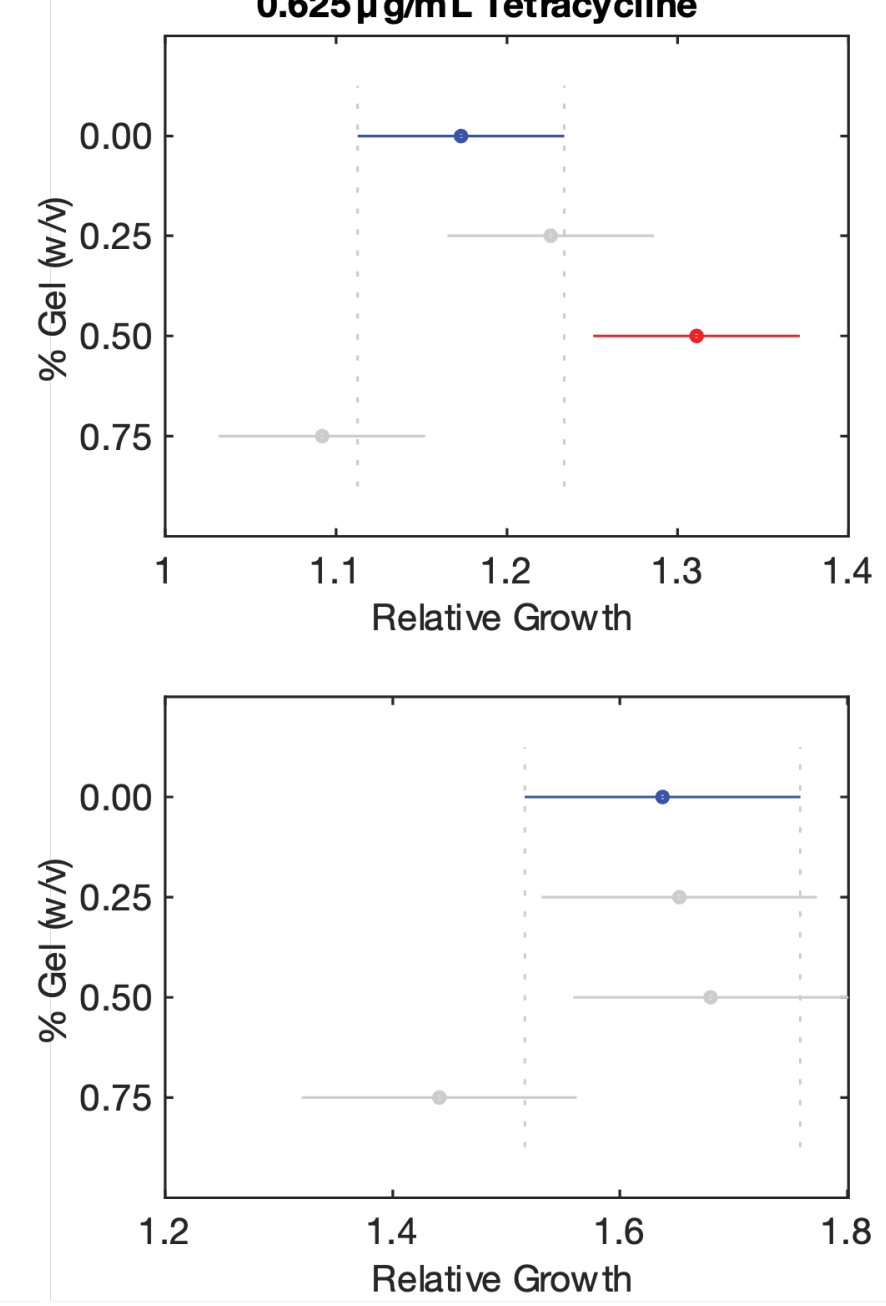


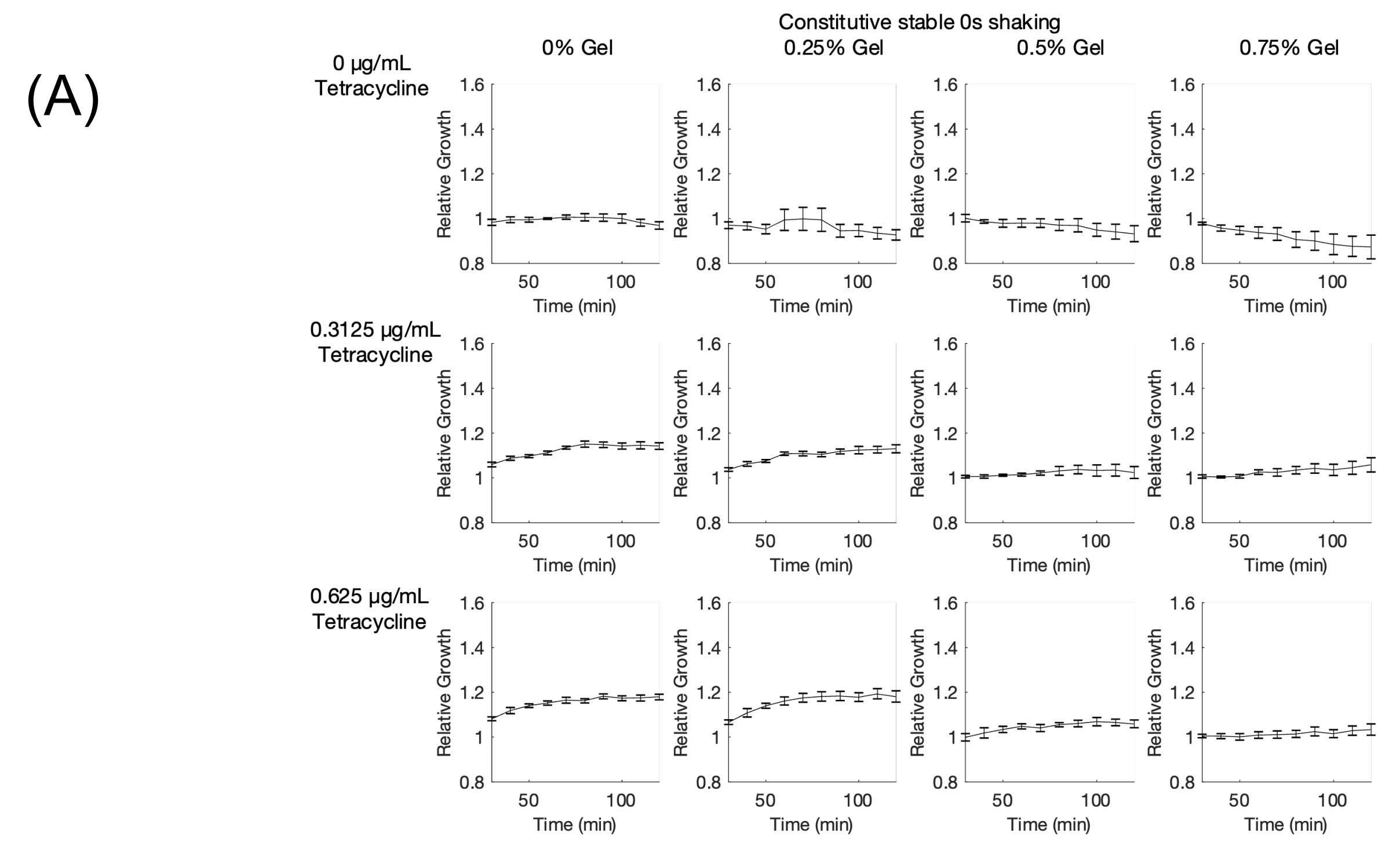




\section{Supplementary Figure 7 - continued}

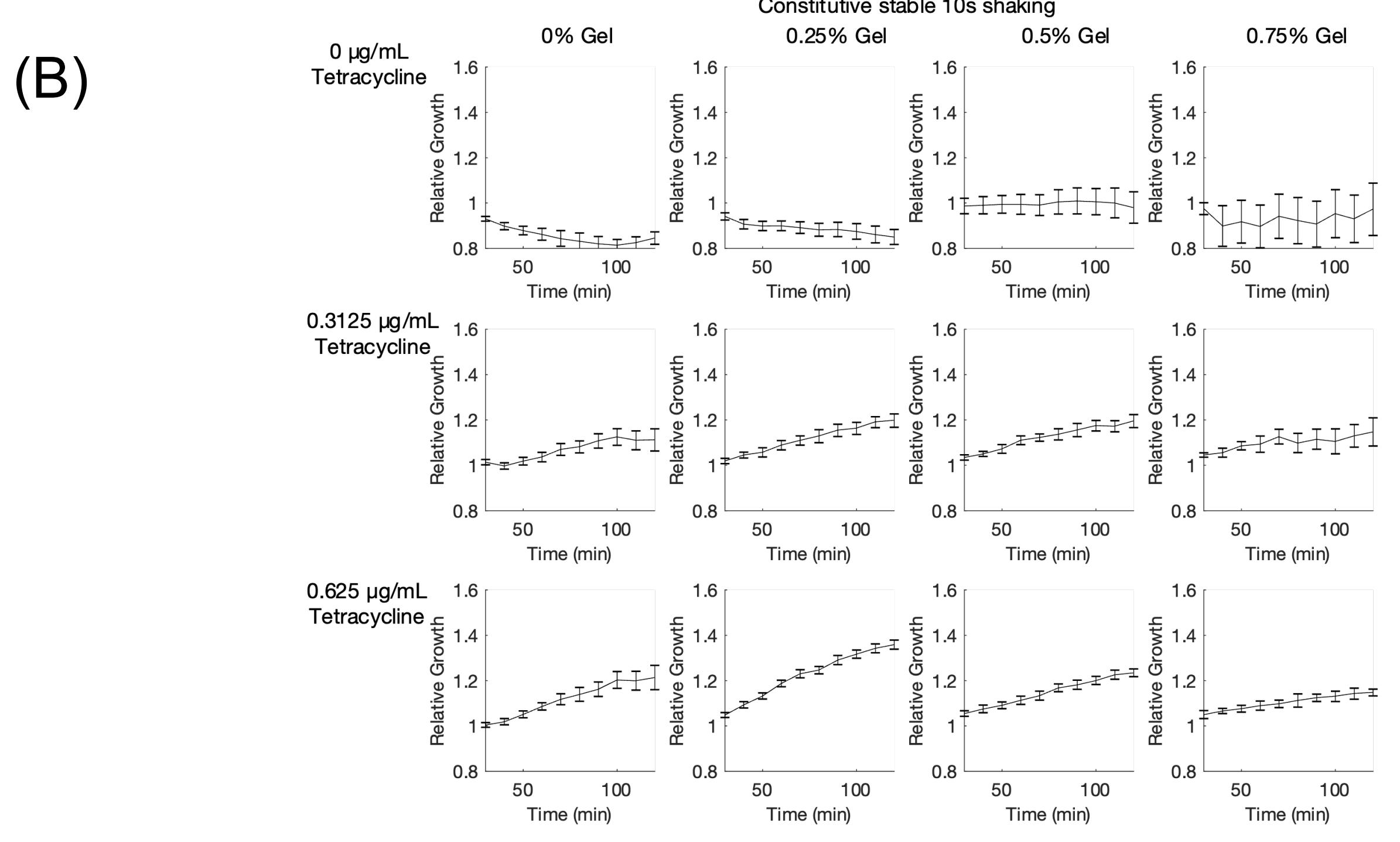




\section{Supplementary Figure 7 - continued}

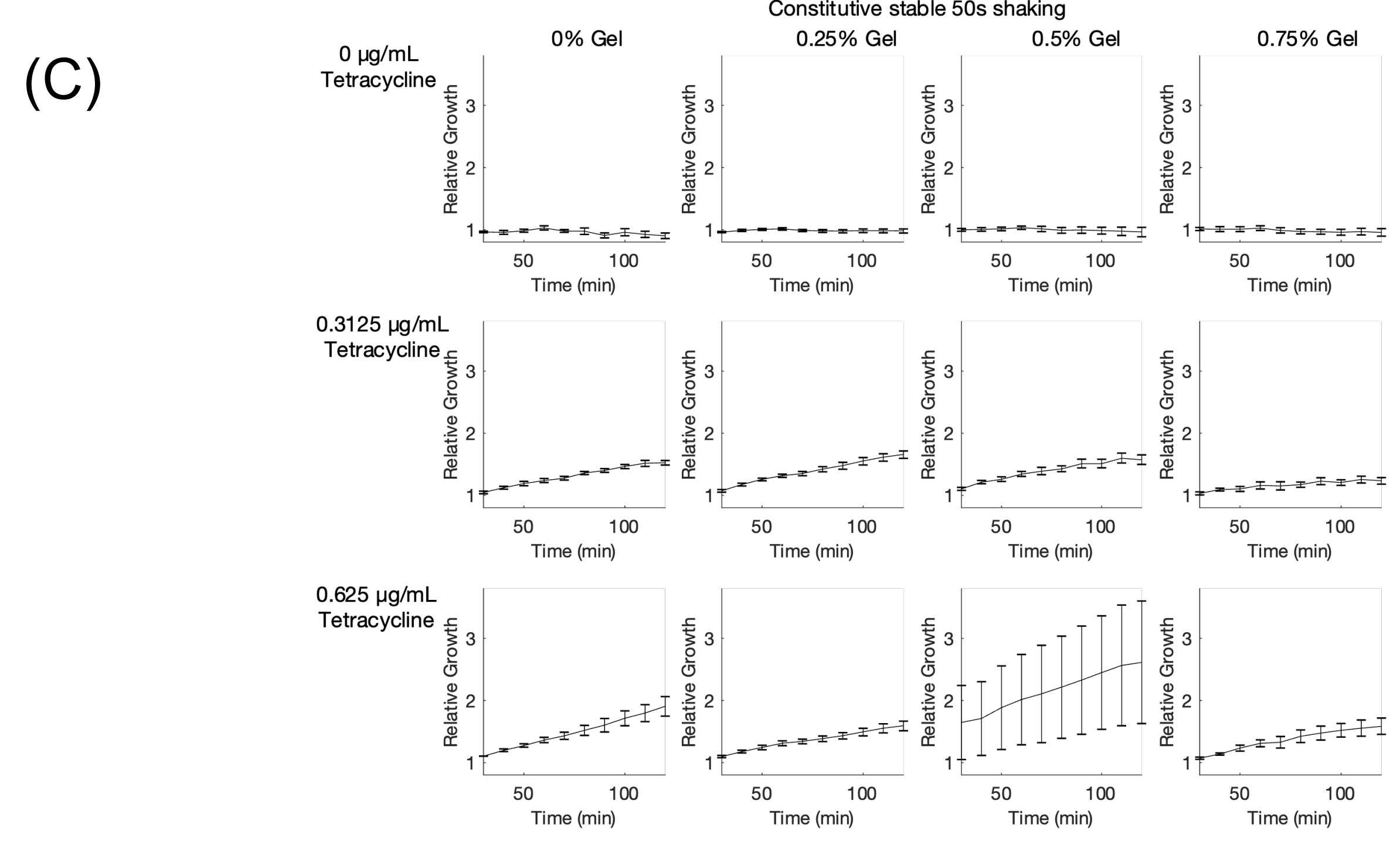




\section{Supplementary Figure 7 - continued}

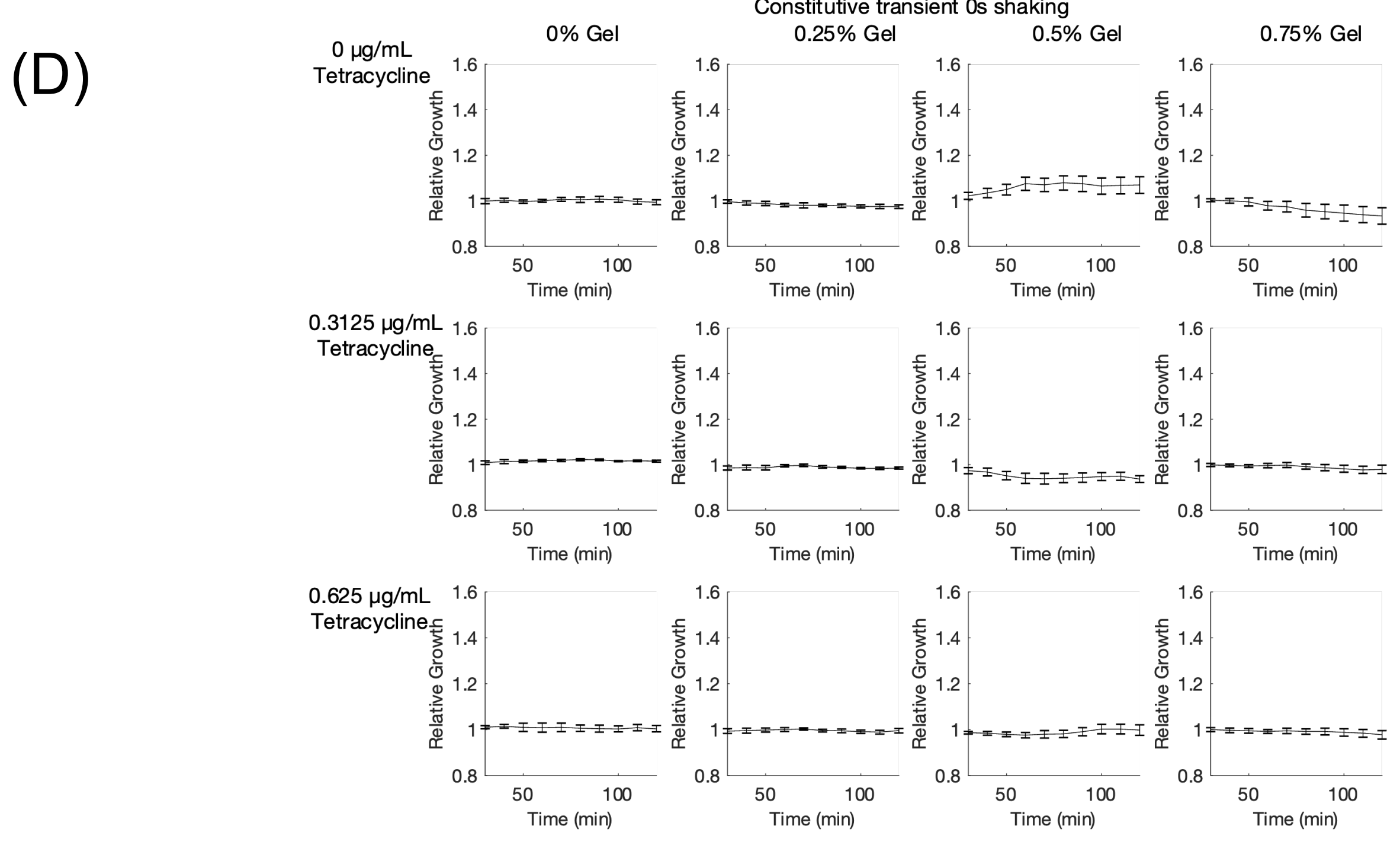




\section{Supplementary Figure 7 - continued}

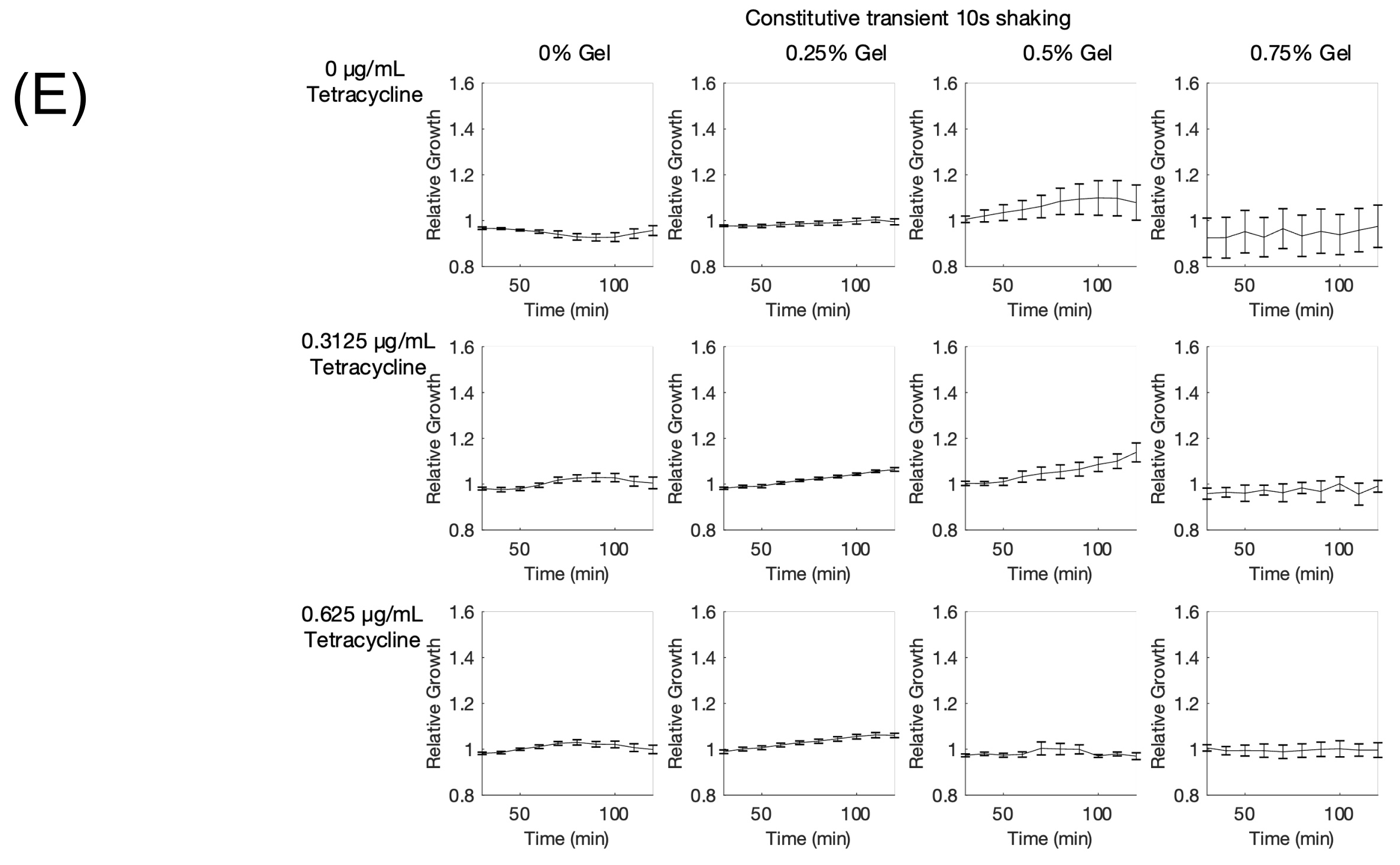


Supplementary Figure 8

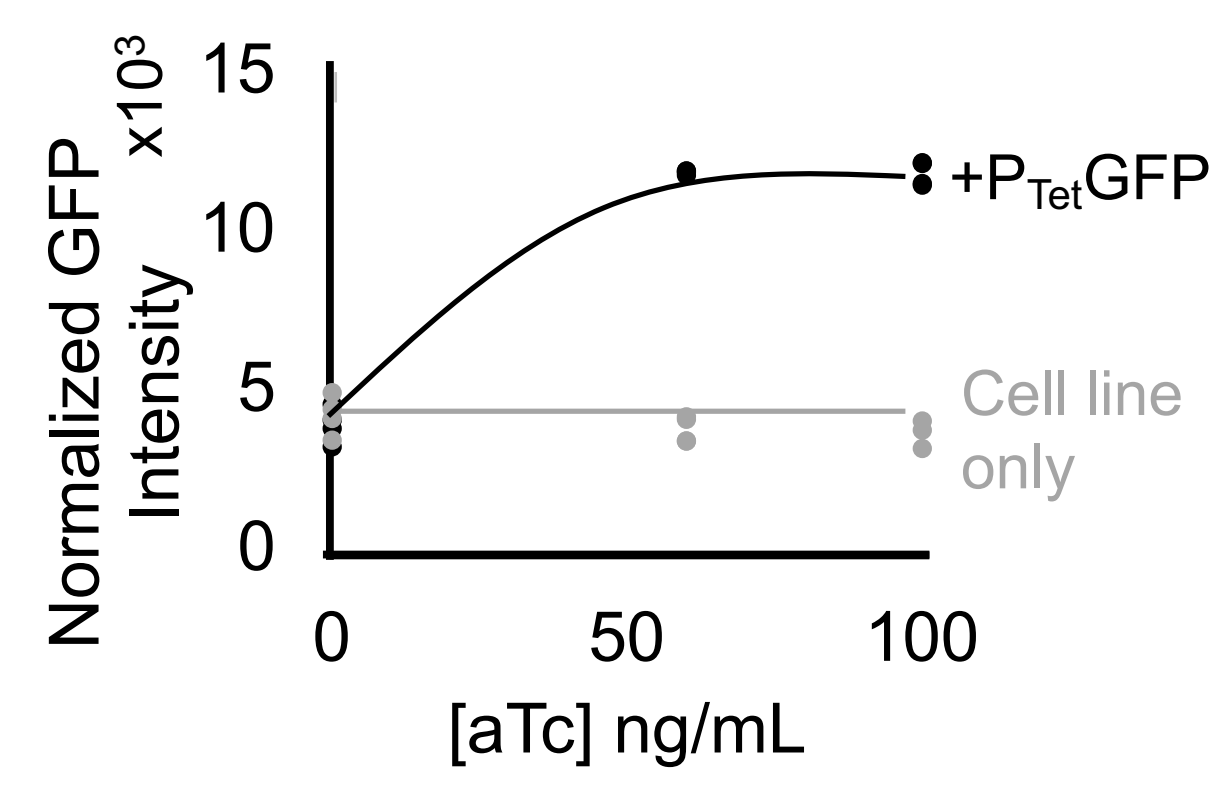




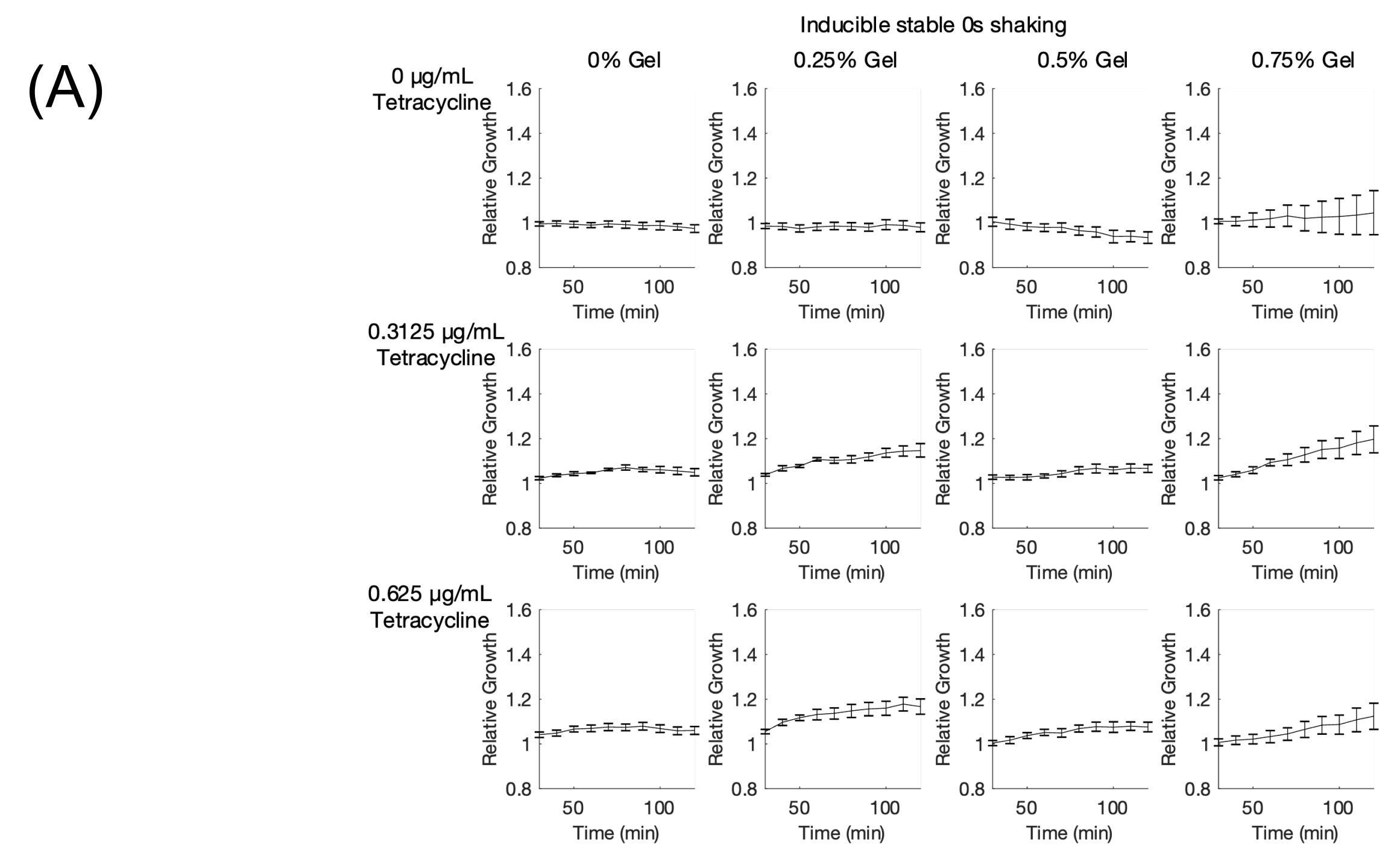




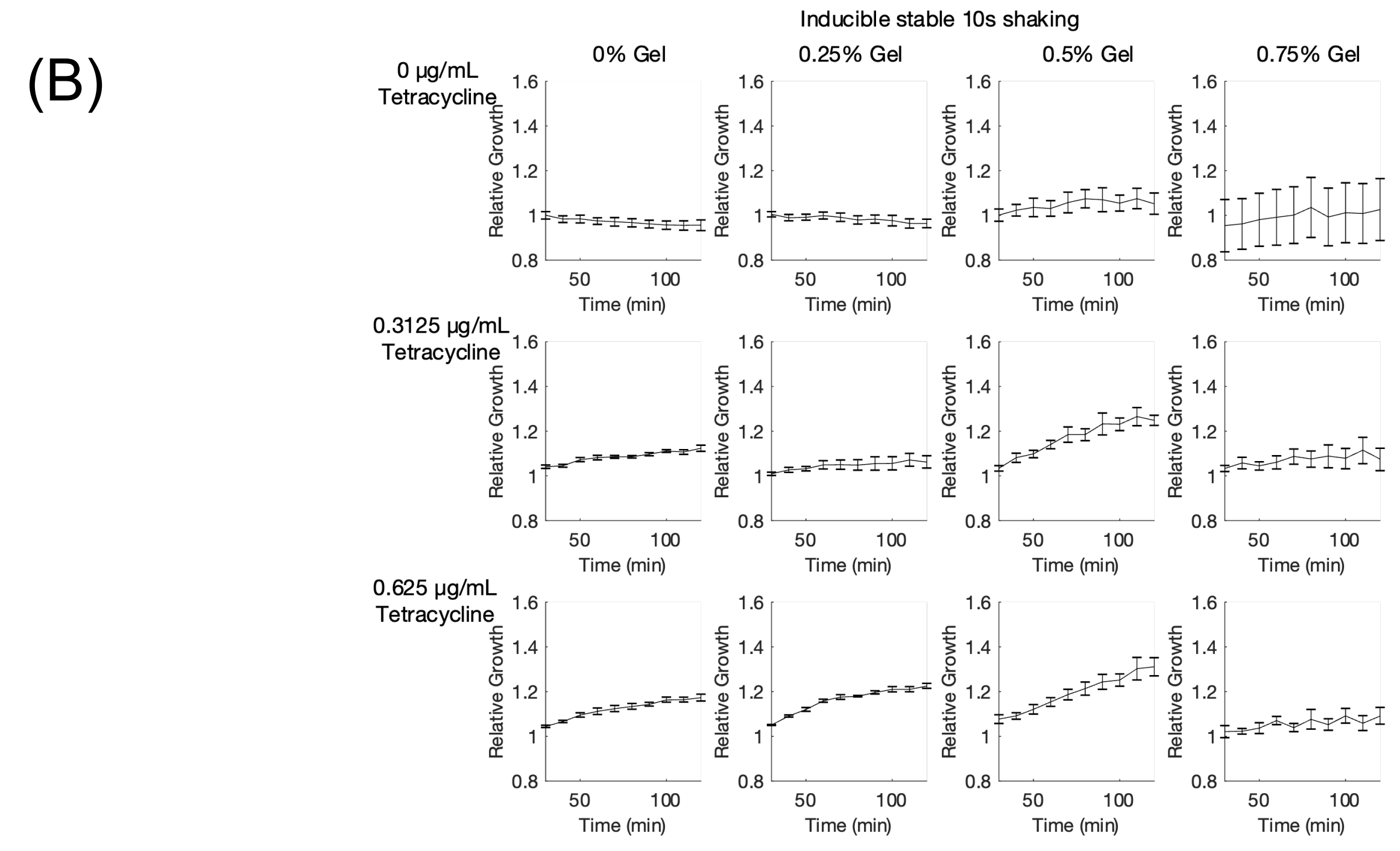




\section{Supplementary Figure 9 - continued}

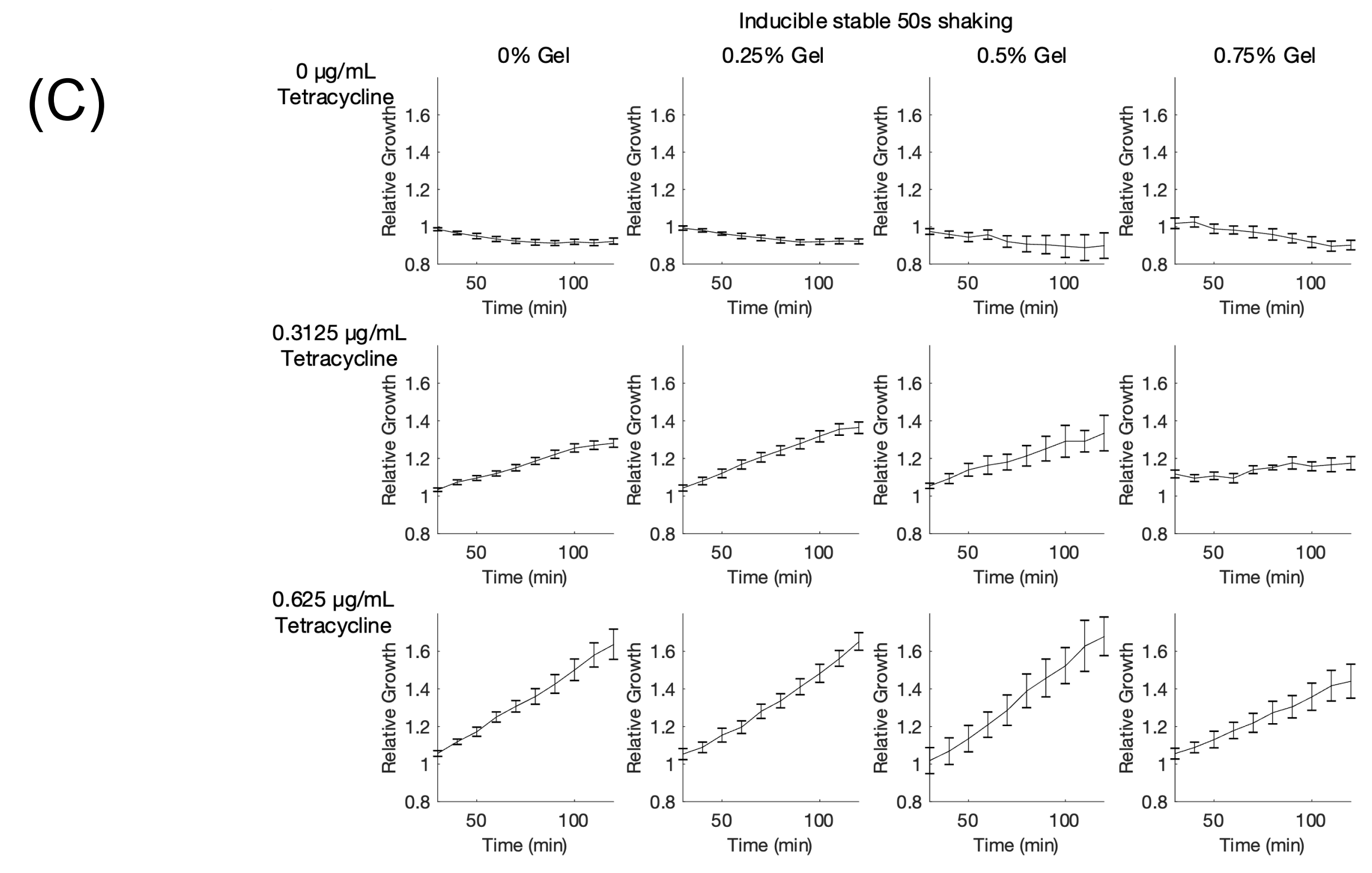




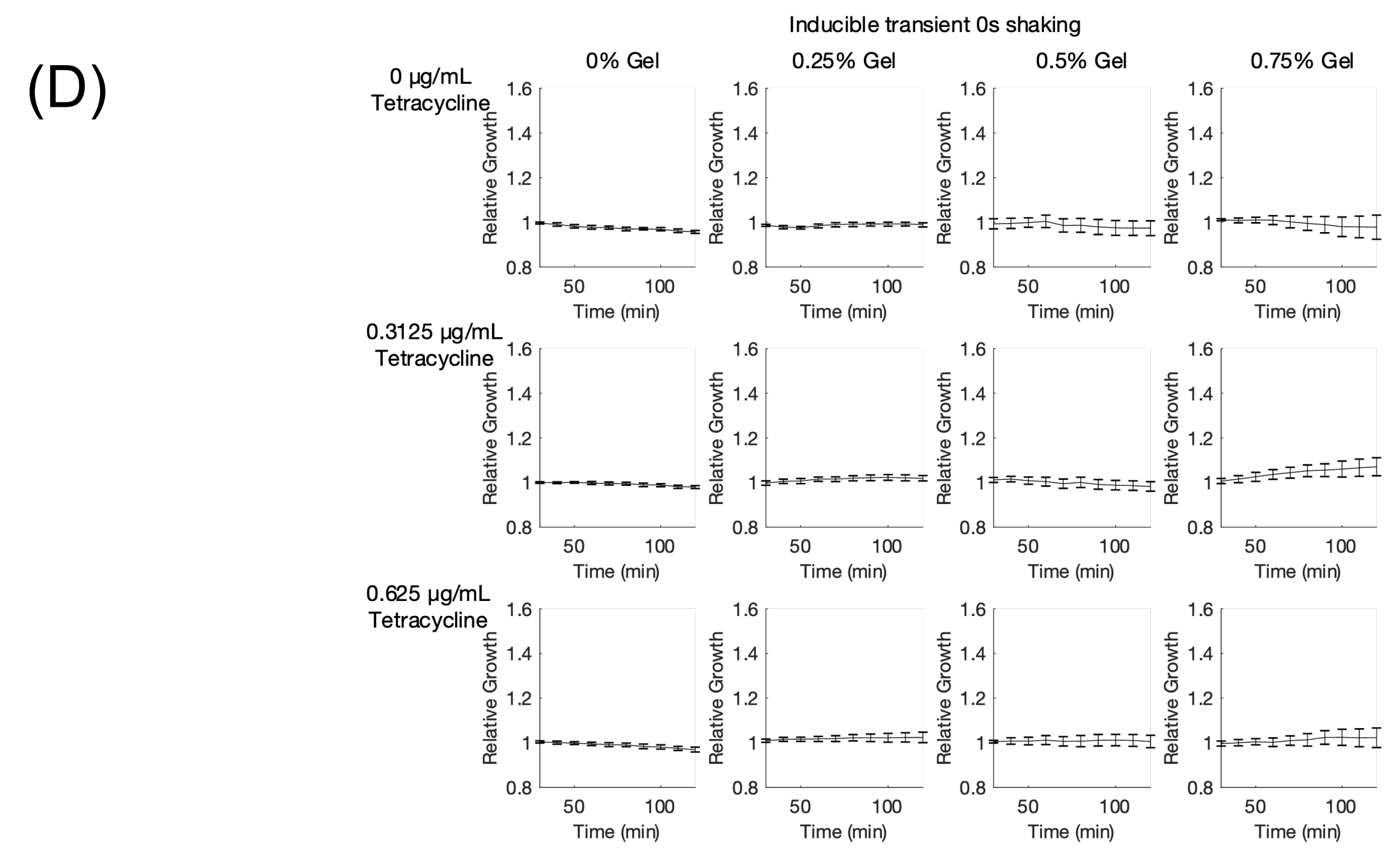




\section{Supplementary Figure 9 - continued}

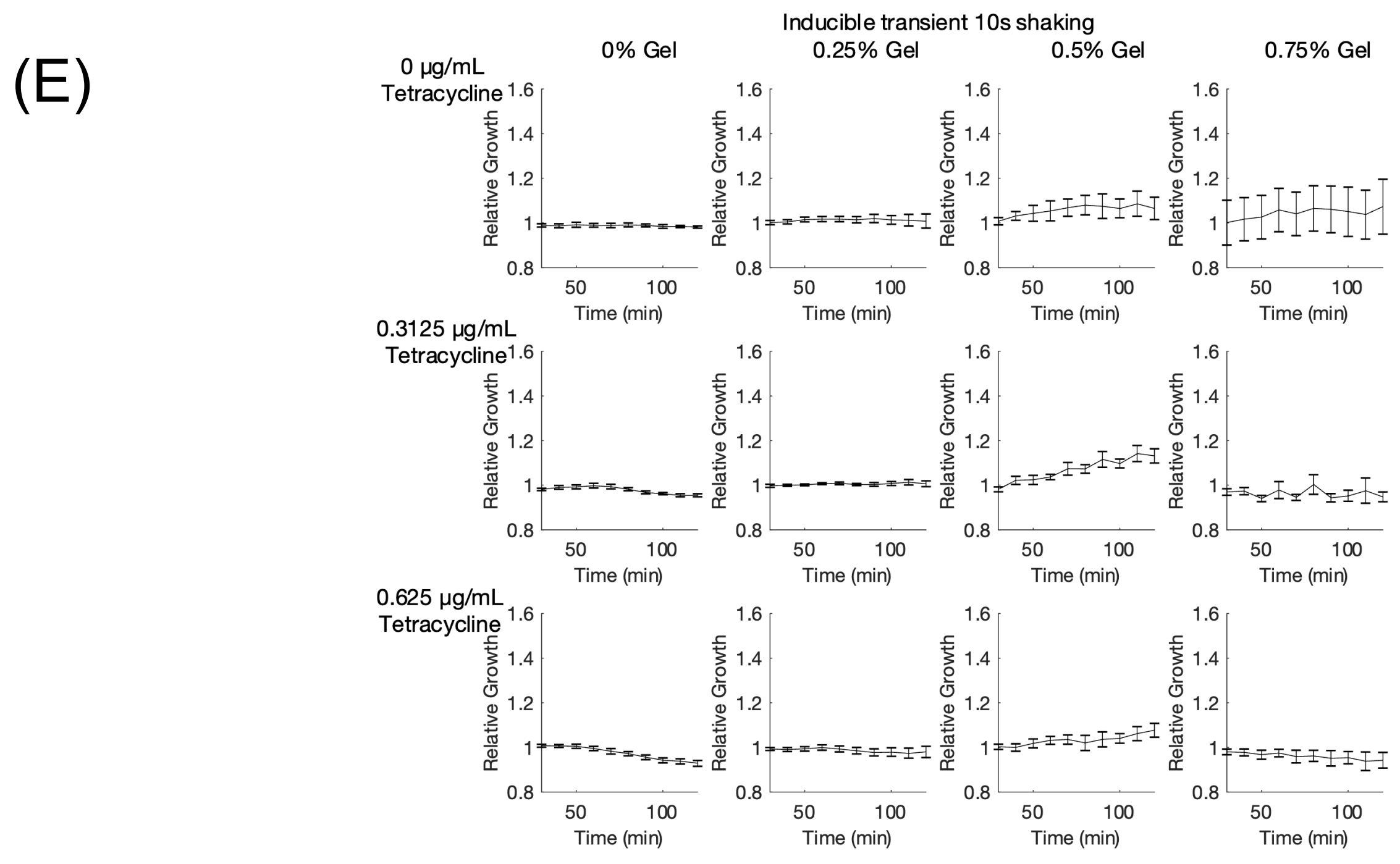

